\title{
Group A Streptococcus intranasal infection promotes CNS infiltration by streptococcal-specific Th17 cells
}

\author{
Thamotharampillai Dileepan, ${ }^{1}$ Erica D. Smith, ${ }^{2}$ Daniel Knowland, ${ }^{2}$ Martin Hsu, ${ }^{2}$ Maryann Platt, ${ }^{3}$ Peter Bittner-Eddy, ${ }^{1}$ \\ Brenda Cohen, ${ }^{1}$ Peter Southern, ${ }^{1}$ Elizabeth Latimer, ${ }^{4}$ Earl Harley, ${ }^{5}$ Dritan Agalliu, ${ }^{2,3}$ and P. Patrick Cleary ${ }^{1}$ \\ 'Department of Microbiology and Immunology, University of Minnesota, Minneapolis, Minnesota, USA. ²Department of Developmental and Cell Biology, University of California, Irvine, California, USA. \\ ${ }^{3}$ Departments of Neurology, Pathology and Cell Biology and Pharmacology, Columbia University Medical Center, New York, New York, USA. ${ }^{4}$ Child and Adolescent Neurology, Department of Pediatrics, \\ and ${ }^{5}$ Department of Otolaryngology, Georgetown University, Washington, DC, USA.
}

\begin{abstract}
Group A streptococcal (CAS) infection induces the production of Abs that cross-react with host neuronal proteins, and these anti-CAS mimetic Abs are associated with autoimmune diseases of the CNS. However, the mechanisms that allow these Abs to cross the blood-brain barrier (BBB) and induce neuropathology remain unresolved. We have previously shown that CAS infection in mouse models induces a robust Th17 response in nasal-associated lymphoid tissue (NALT). Here, we identified GAS-specific Th17 cells in tonsils of humans naturally exposed to CAS, prompting us to explore whether CAS-specific CD4+ T cells home to mouse brains following i.n. infection. Intranasal challenge of repeatedly CAS-inoculated mice promoted migration of CAS-specific Th17 cells from NALT into the brain, BBB breakdown, serum IgC deposition, microglial activation, and loss of excitatory synaptic proteins under conditions in which no viable bacteria were detected in CNS tissue. CD4 ${ }^{+} \mathrm{T}$ cells were predominantly located in the olfactory bulb (OB) and in other brain regions that receive direct input from the $\mathrm{OB}$. Together, these findings provide insight into the immunopathology of neuropsychiatric complications that are associated with GAS infections and suggest that crosstalk between the CNS and cellular immunity may be a general mechanism by which infectious agents exacerbate symptoms associated with other CNS autoimmune disorders.
\end{abstract}

\section{Introduction}

Pharyngitis caused by Streptococcus pyogenes (group A Streptococcus [GAS]) is a common, treatable infection; however, autoimmune sequelae associated with GAS infections, including rheumatic fever and rheumatic heart disease as well as motor and neuropsychiatric disorders, can produce chronic disability (1). Sydenham chorea (SC) is characterized by uncoordinated motor involvement and is reported to occur in $20 \%$ to $30 \%$ of children with acute rheumatic fever $(2,3)$. An increasingly recognized neuropsychiatric risk, pediatric autoimmune neuropsychiatric disorders associated with streptococcal infections (PANDAS) affect a subset of individuals with abrupt onset of obsessive-compulsive disorder (OCD), anorexia nervosa, separation anxiety, and other abnormal behaviors (3-7). Subsequent episodes of OCD are often associated with GAS infections or other undefined triggers. The time of onset and periodic exacerbation of symptoms, positive responses to immune therapy, and discovery of autoantibodies are all consistent with an autoimmune mechanism; yet the role of $T$ cells and the route of autoantibody entry into the CNS in SC and PANDAS remain to be defined $(6,8-10)$.

The connection between GAS infection, neuronal-specific autoantibodies, and SC is well established; however, the link

Authorship note: T. Dileepan and E.D. Smith contributed equally to this work. D. Agalliu and P.P. Cleary are co-senior authors and contributed equally to this work.

Conflict of interest: The authors have declared that no conflict of interest exists. Submitted: January 5, 2015; Accepted: November 5, 2015.

Reference information: J Clin Invest. 2016;126(1):303-317. doi:10.1172/JCI80792. between infection and initial onset or subsequent exacerbations of PANDAS is still debated. Indeed, very little is known about CNS immunopathology associated with bacterial infections, although $\mathrm{T}$ cell responses to viral encephalitis (11) and the T cell-mediated immunopathology of multiple sclerosis (MS) are well characterized (12). Behavioral changes and IgG deposition in the brain have been reported in mouse (13) and rat (14) models following administration of serum from animals immunized with heat-killed GAS (HK-GAS) or immunization with bacterial protein extracts. Yet, the mechanism by which Abs cross the blood-brain barrier (BBB) in rodents is unknown, because development of behavioral deficits in these models required coadministration of either Bordetella pertussis toxin or LPS, two agents that disrupt the BBB $(13,15)$.

GAS has a tropism for murine nasal-associated lymphoid tissue (NALT), which is functionally equivalent to human palatine tonsils (16). Recurrent GAS i.n. infections in mice induce a dominant, TGF- $\beta 1$ - and IL-6-dependent, protective Th17 cellular response in NALT. Repeated i.n. infections expand Th17 cells and shift their cytokine profile to one that is $\operatorname{IL}-17 \mathrm{~A}^{+} \operatorname{IFN}-\gamma^{+}(17,18)$. IL-17A can disrupt BBB function in vitro and in vivo through the generation of ROS in endothelial cells $(19,20)$. Moreover, IL-17A ${ }^{+}$ and IL-17A $\mathrm{A}^{+} \mathrm{IFN}-\gamma^{+}$double-positive Th cells are known to home to the CNS in both human MS and rodent models of the disease (21). Peripheral blood contains few IL-17A $\mathrm{A}^{+} \mathrm{T}$ cells; however, tonsils are reported to contain large numbers of $\mathrm{CD} 4^{+} \mathrm{IL}-17 \mathrm{~A}^{+} \mathrm{T}$ cells with unknown antigenic specificity (22). The high incidence of GAS infections in children led us to examine whether tonsils contain streptococcus-specific Th17 cells. Here, we report that human ton- 
A

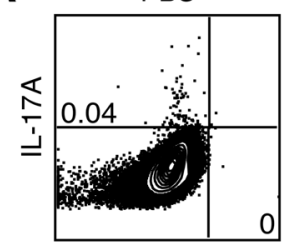

HK-GAS

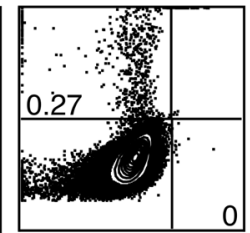

IFN- $\gamma$

C
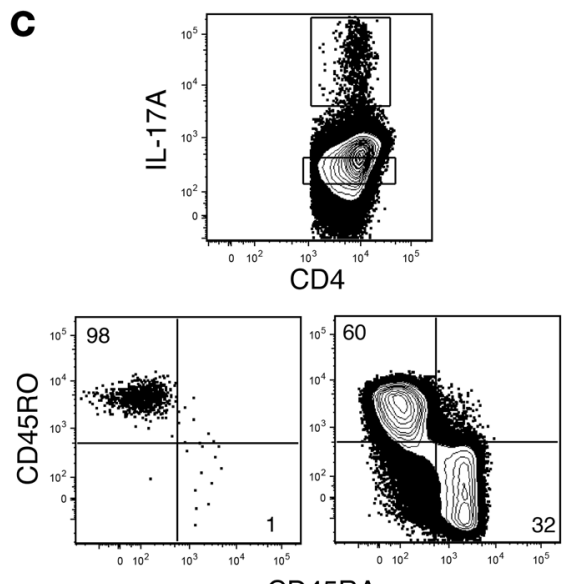

CD45RA

PMA+1

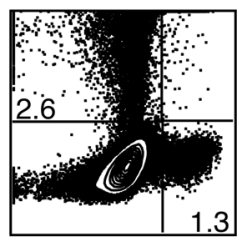

1.3
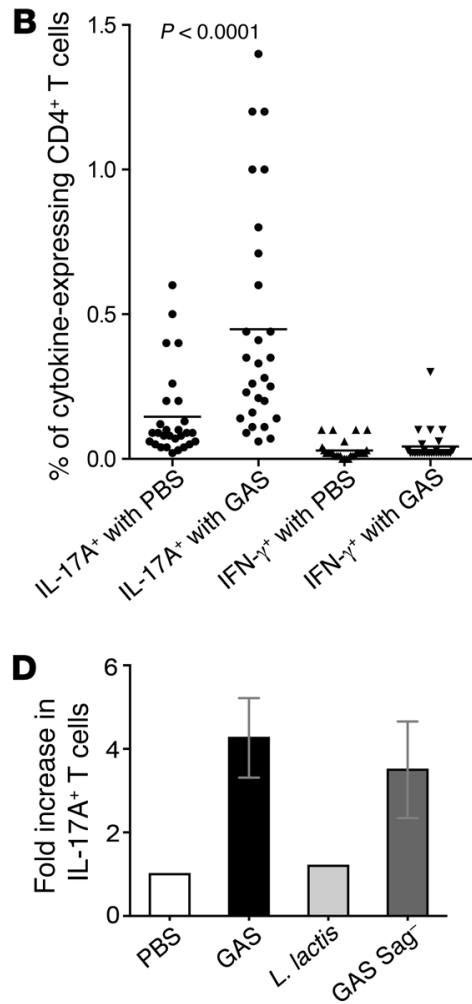

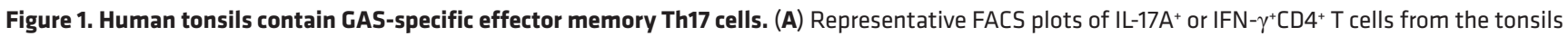
of 1 patient. Single-cell suspensions were incubated for 6 hours with PBS (control), HK-GAS, or PMA+I prior to analysis. Numbers within panels indicate the percentages of total $C D 4^{+} T$ cells that expressed specific cytokines. (B) Scatter plot of cytokine expression as a percentage of total CD4 ${ }^{+} T$ cells analyzed in tissues from 28 patients. Each dot represents an individual patient. Horizontal lines indicate the mean percentage of cytokine-producing cells. Statistical significance of mean differences was assessed with the Wilcoxon matched-pairs signed-rank test. Data are representative of 10 experiments. (C) FACS plots for HK-CAS-activated tonsil cells stained for IL-17A, CD4, CD45RO, and CD45RA. Cells shown at the top of the IL-17A/CD4 panel and in the left panel for CD45R0/CD45RA were gated on IL-17A+CD4+ $T$ cells, and cells at the bottom of the IL-17A/CD4 panel and in the right panel for CD45RO/CD45RA were gated on IL-17A-CD4+ $T$ cells. (D) Bar graph shows the fold increase in CD4+IL-17A+ cells after single-cell suspensions from 1 patient were incubated with PBS (white), HK-CAS (black), L. lactis (light gray), or HK-CAS, a Sag- strain that lacks all known superantigen genes (23) (dark gray). Bar graphs show the mean \pm SEM.

sils contain large numbers of GAS-specific Th17 cells. The proximity of mucosal lymphoid tissues to the cribriform plate, coupled with our discovery of significant numbers of GAS-specific T cells in human tonsils, prompted us to investigate whether immunization by multiple i.n. streptococcal infections promotes bacterium-specific Th17 cells to enter the brain in mice. Our results indicate the presence in the brain of GAS-specific Th17 cells, which are accompanied by alterations in BBB integrity that enable serum IgG deposition, neuroinflammation (microglia activation), and deficits in synaptic connectivity.

\section{Results}

Human tonsils are populated with GAS-specific Th17 cells. We first examined whether tonsils from children contain streptococcusspecific Th17 cells. Single-cell suspensions of human tonsils from 28 patients were stimulated with either PBS, heat-killed streptococci (HK-GAS), or PMA and ionomycin (PMA+I) for 6 hours ex vivo before $\mathrm{T}$ cell markers and intracellular cytokine expression were evaluated by FACS. Similar to data reported by Lim et al. (22), incubation of single-cell suspensions from tonsils with PMA+I showed that $0.2 \%$ to $5.1 \%$ and $0.04 \%$ to $3.1 \%$ of $\mathrm{CD}^{+} \mathrm{T}$ cells produced either IL-17A or IFN- $\gamma$, respectively (a representative speci- men is shown in Figure 1A, right panel). A smaller but substantial number of T cells expressed both IL-17A and IFN- $\gamma$. Incubation of the same specimens with HK-GAS induced IL-17A expression in a significant $(\mathrm{P}<0.0001)$ number of $\mathrm{CD} 4^{+} \mathrm{T}$ cells; however, we found that very few cells expressed either IFN- $\gamma$ or both IL-17A and IFN- $\gamma$ (Figure 1A, middle panel). Overall, in tissues collected from 28 individuals, the mean frequencies of $\mathrm{CD}_{4}^{+} \mathrm{T}$ cells that expressed IL-17A after incubation with GAS (0.45\%) were significantly greater than those seen in control tissues incubated with PBS (0.14\%) (Figure 1B). In order to account for differences in cell viability from various specimens, the percentages of $\mathrm{CD} 4^{+} \mathrm{T}$ cells that expressed IL-17 or IFN- $\gamma$ after incubation with GAS were normalized against cell populations activated with PMA+I (Supplemental Figure 1A; supplemental material available online with this article; doi:10.1172/JCI80792DS1). This analysis confirmed the presence of a GAS-induced, antigen-specific Th17 cell population compared with PBS controls $(P<0.0001)$. Our assessment of other $\mathrm{T}$ cell markers revealed that more than $90 \%$ of GAS-specific Th17 cells from human tonsils expressed high levels of CD45RO and low levels of CD45RA (Figure 1C), CCR7, and CD62L markers (Supplemental Figure 1B), consistent with an effector memory $\mathrm{T}$ cell phenotype. In order to further evaluate antigen specificity of 
A

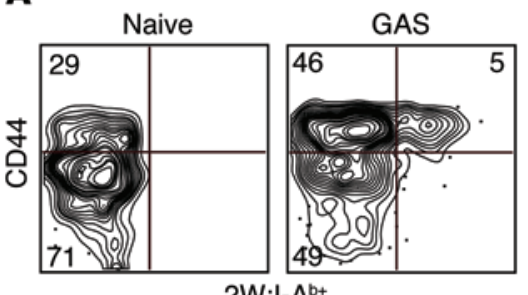

$2 W: I-A^{b+}$

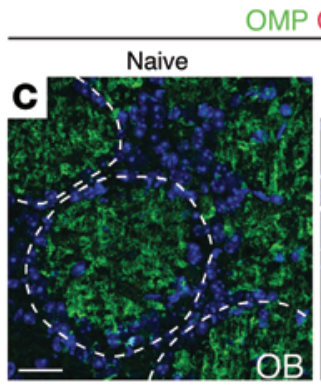

MP CD4 DAPI

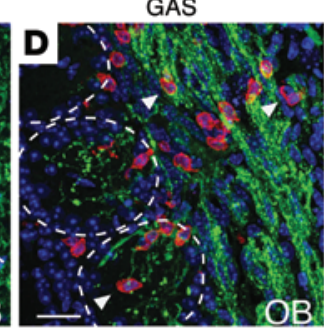

CD4 Caveolin 1
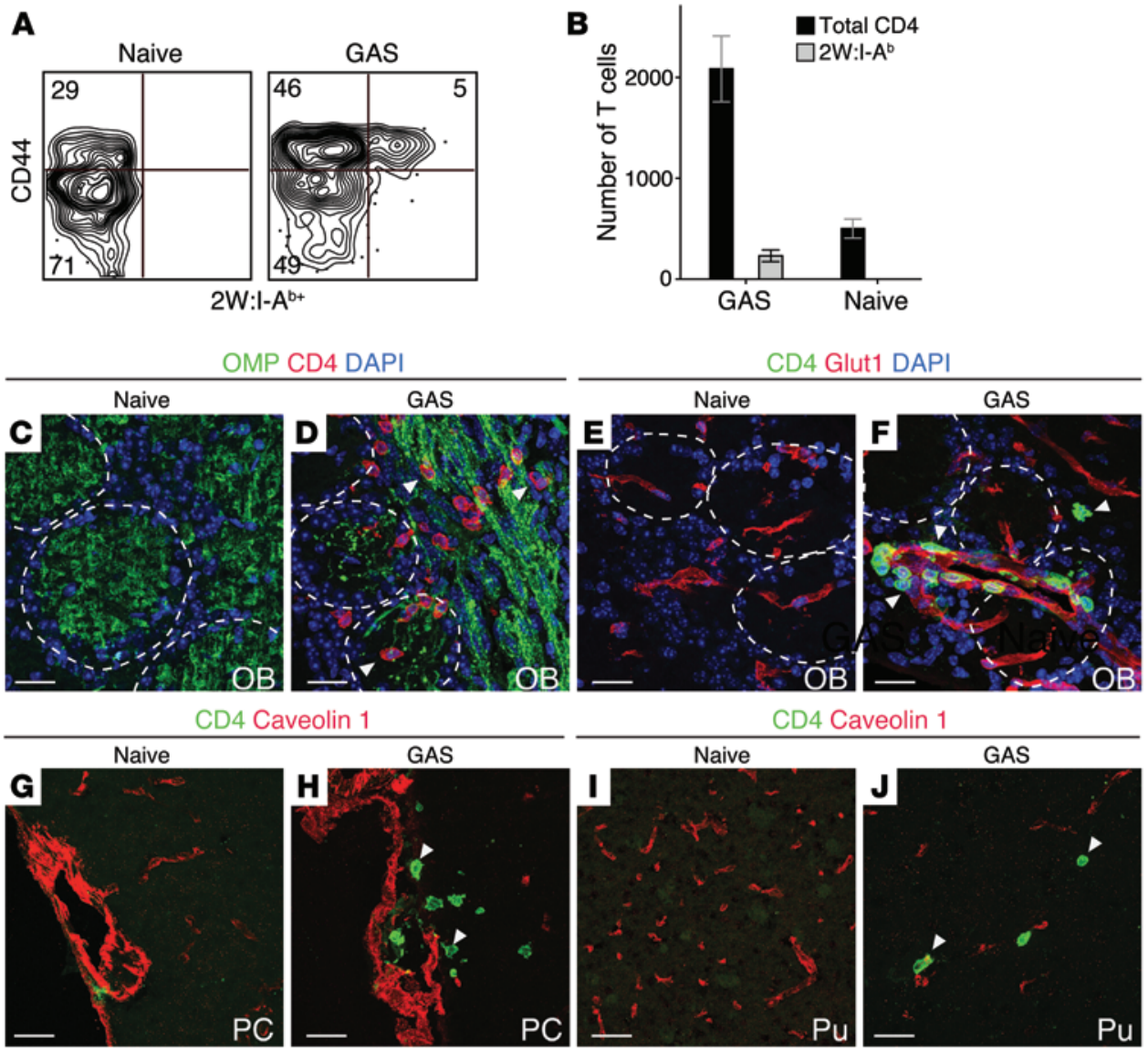

CD4 Caveolin 1
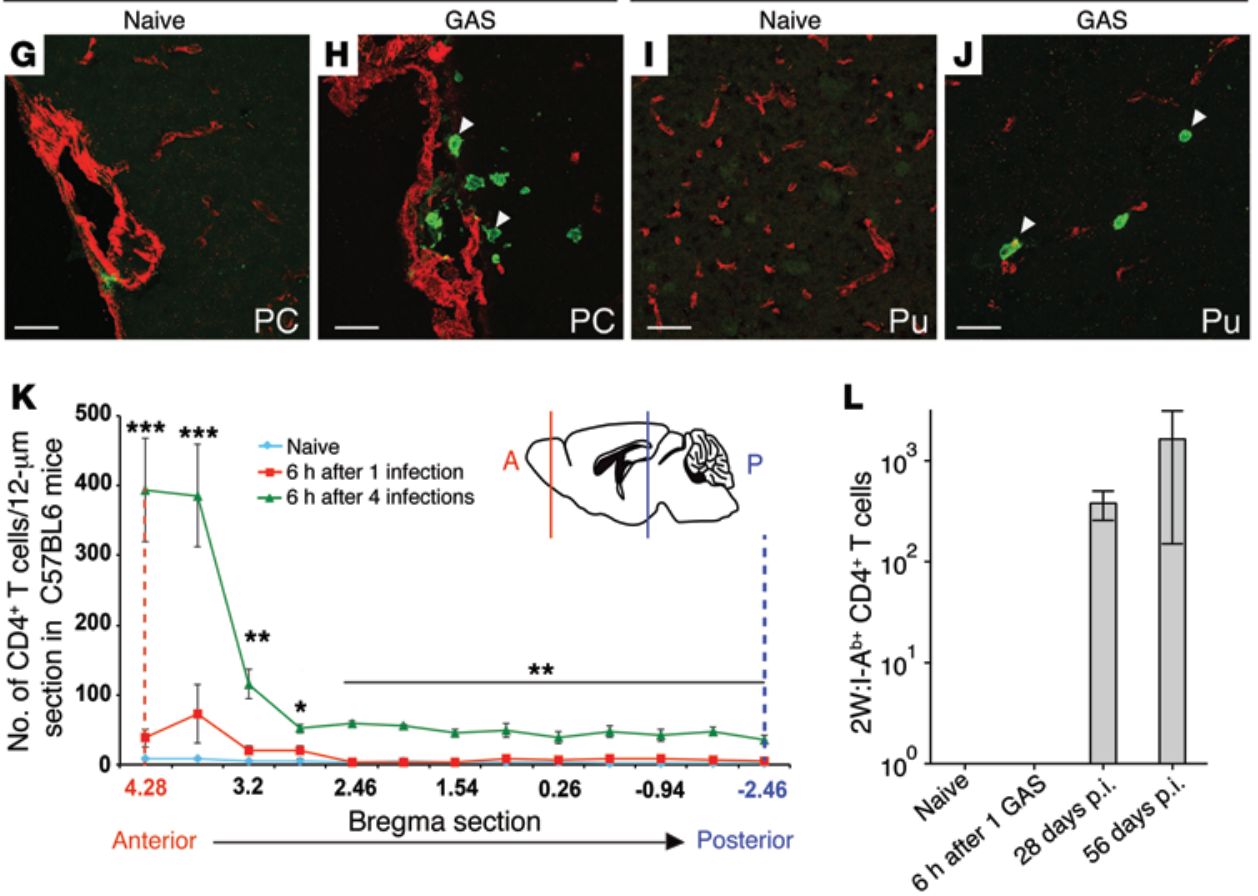

Figure 2. Immunization of mice by multiple i.n. inoculations generates predominantly GAS-specific CD4 ${ }^{+} \mathrm{T}$ cells that home to and persist in the brain. (A) Representative FACS plot of $\mathrm{CD}^{+} \mathrm{T}$ cells isolated from the brains of naive or multiply GAS-inoculated mice 48 hours after the last inoculation. (B) Bar graph showing total (black) or GAS-specific 2W:I-A ${ }^{\text {b+ }}$ (gray) CD4+ T cells from the brains of GAS-inoculated $(n=4)$ and naive mice $(n=2)$. (C-F) CD4 $T$ cells (red) are distributed in the outer nerve (olfactory marker protein [OMP], green) and glomerular (DAPI, dashed white lines) layers of OBs in CASinoculated animals (D). White arrowheads point to $T$ cells that colocalized with OMP (green). T cells also associated with Glut $1^{+}$blood vessels (red) in GAS-inoculated mice (F). (G-J) CD4+ T cells were found in the $P C$ and putamen (Pu) in GAS-inoculated ( $\mathbf{H}$ and $\mathbf{J}$ ), but not naive, mice (G and I). Anti-caveolin 1 (red) labels blood vessels. (K) Line graph of $\mathrm{CD4}^{+} \mathrm{T}$ cell distribution along the anteroposterior axis of the brain in multiply (green) or singly CAS-inoculated (red) or naive (aqua) mice. The $x$ axis represents the bregma region, and the $y$ axis shows the number of $C D 4^{+} \mathrm{T}$ cells per $12-\mu \mathrm{m}$ section. Solid red and blue lines indicate positions in the brain relative to the graph (dashed lines). Data were collected from 3 independent experiments for 3 to 8 animals per group and are presented as the mean \pm SEM. ${ }^{*} P<0.05$, ${ }^{* *} P<0.01$, and ${ }^{* * *} P<0.001$, by 2-way ANOVA with Bonferroni's posthoc correction. (L) Bar graph of GASspecific ( $\left.2 \mathrm{~W}: \mathrm{I}-\mathrm{A}^{\mathrm{b}+}\right) \mathrm{CD} 4^{+} \mathrm{T}$ cells isolated and FACS sorted from the brains of naive $(n=2)$ and CAS-2W-inoculated mice 6 hours after 1 i.n. infection $(n=2)$ and 28 days $(n=4)$ or 56 days $(n=2)$ after 5 i.n. inoculations. p.i., post infection. Bar ( $\mathbf{B}$ and $\mathbf{L}$ ) and line ( $\mathbf{K})$ graphs show the mean \pm SEM. Scale bars: $50 \mu \mathrm{m}(\mathbf{C}-\mathbf{J})$.
IL-17A expression, single-cell human tonsil suspensions were incubated with either Lactococcus lactis, a Gram-positive bacterium that expresses TLR ligands similar to those expressed by GAS, or SagGAS, a strain that lacks all known superantigen genes (23). Incubation of tonsils with heat-killed L. lactis did not induce expression of IL-17A by T cells; however, incubation with heat-killed Sag- GAS activated T cells to secrete IL-17A (Figure 1D). Although we cannot completely rule out some involvement of TLR-mediated activation of T cells by GAS, these findings strongly suggest that GAS-dependent activation of IL-17A expression by T cells from human tonsils is largely antigen specific.

Multiple i.n. GAS infections in mice induce GAS-specific CD $4^{+}$ $T$ cells that home to the brain. The association of Th17 cells with several autoimmune diseases and their presence in both human ton- sils (Figure 1 and Supplemental Figure 1) and murine NALT after i.n. infection (17) prompted us to investigate whether these cells migrate to the brain. Epitope-tagged streptococci-specific (GAS$2 \mathrm{~W}$-specific) $\mathrm{T}$ cells were induced in $\mathrm{C} 57 \mathrm{BL} / 6$ mice by repeated i.n. inoculations with $2 \times 10^{8} \mathrm{CFU}$ bacteria over a 5 -week period $(17,18)$. After perfusion with PBS, leukocytes were purified from the brains, NALT, and cervical lymph nodes (CLNs) from both GAS-2W-inoculated and control naive mice, then restimulated ex vivo with $\mathrm{PMA}+\mathrm{I}$ in order to assess cytokine expression profiles. $2 \mathrm{~W}$ is a variant peptide that consists of amino acids $52-68$ from an I-E $\alpha$ MHCII protein, which is expressed by GAS-2W as an N-terminal fusion to $\mathrm{M} 1$ protein (17). $\mathrm{CD}^{+} \mathrm{T}$ cells that express $\mathrm{T}$ cell receptors specific for the $2 \mathrm{~W}$ peptide bound to MHCII complex $\mathrm{I}-\mathrm{Ab}$ (2W:I-A ${ }^{\mathrm{b}}$-specific $\mathrm{T}$ cells) were detected by staining cells with 
A
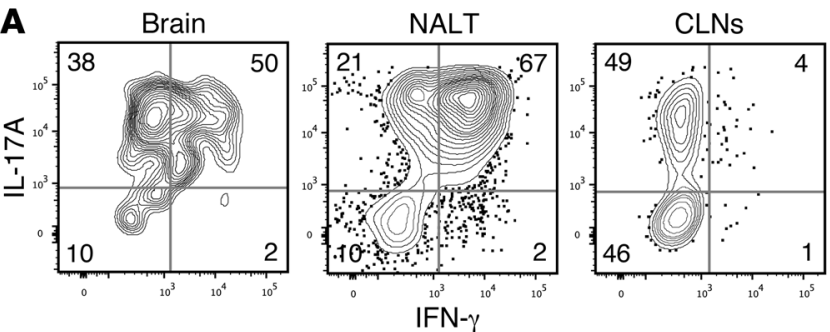

B
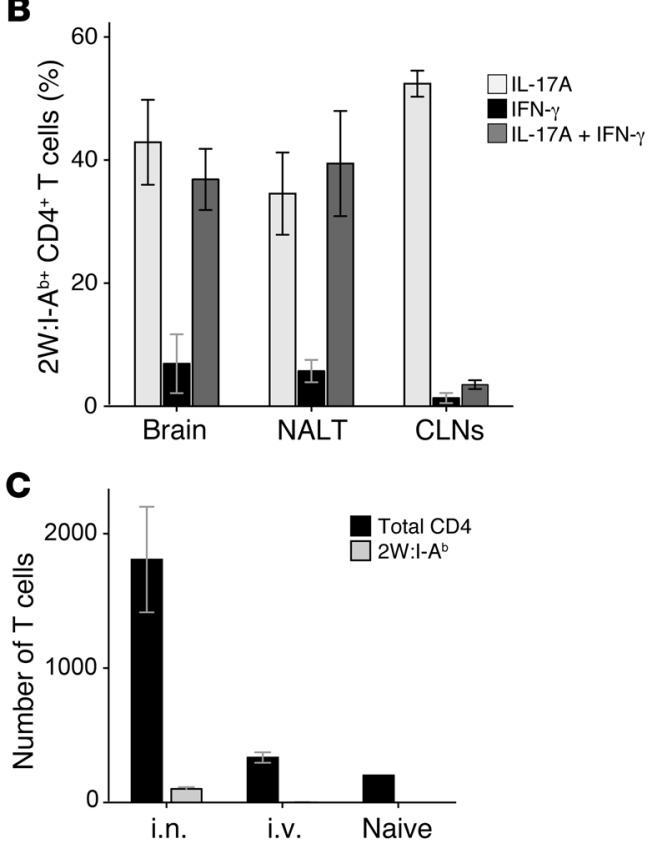

D

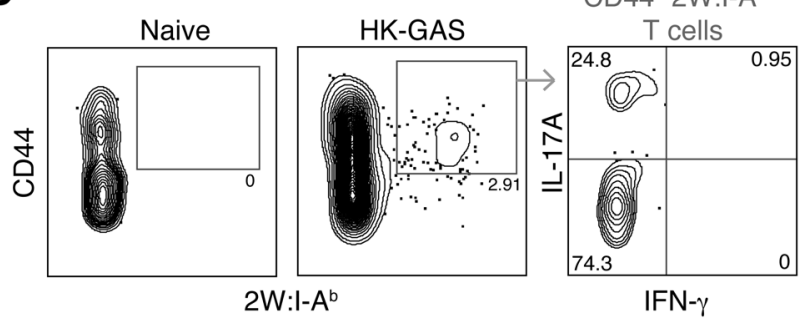

fluorochrome-labeled $2 \mathrm{~W}: \mathrm{I}-\mathrm{A}^{\mathrm{b}}$ tetramers as previously described (24). GAS-inoculated mice had higher numbers of $\mathrm{CD}^{+}$GASspecific (2W:I-A $\left.{ }^{\mathrm{b}+}\right)$ T cells in their brains than did uninfected controls (Figure 2, A and B). More than $50 \%$ of $\mathrm{CD}^{+} \mathrm{T}$ cells expressed high levels of CD44, indicating previous experience with cognate antigens, and approximately $10 \%$ of those cells were $2 \mathrm{~W}: \mathrm{I}-\mathrm{A}^{\mathrm{b}}$ positive. As expected, no $\mathrm{CD} 4^{+} 2 \mathrm{~W}: \mathrm{I}^{-\mathrm{A}^{\mathrm{b}+}} \mathrm{T}$ cells were detected in tissue from naive mice (Figure 2, A and B). Brains from mice that were inoculated i.n. multiple times with GAS also contained high numbers of $\mathrm{CD} 4{ }^{+} \mathrm{CD} 44^{\text {hi }} 2 \mathrm{~W}: \mathrm{I}-\mathrm{A}^{\mathrm{b}-\mathrm{T}} \mathrm{T}$ cells, which likely represent $\mathrm{T}$ cells directed against other streptococcal antigens. The few $\mathrm{CD}^{+}$

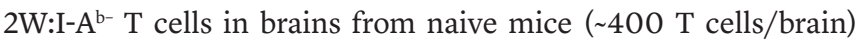
were $\mathrm{T}$ cells we found in the vasculature that were not completely removed by perfusion (see below).

We used immunofluorescence microscopy to analyze the distribution of $\mathrm{CD}^{+} \mathrm{T}$ cells along the anteroposterior axis of the brains of multiply GAS-inoculated mice. $\mathrm{CD}^{+} \mathrm{T}$ cells were pri-
Figure 3. Streptococcus-specific brain-derived T cells express cytokines similar to those derived from NALT. (A) Representative FACS plots and (B) bar graphs showing cytokine profiles and the distribution of $2 \mathrm{~W}: \mathrm{I}^{-\mathrm{A}^{\mathrm{b}}}$ CD4 ${ }^{+} T$ cells isolated from brain, NALT, or CLNs from GAS-2W i.n. inoculated C57BL/ 6 mice. Data were collected from 3 independent experiments for 4 animals and represent the mean \pm SEM. (C) Bar graphs showing the the total number (CD4+; black) and bacterium-specific (2W:I-A ${ }^{\text {b+ }}$ CD4 $4^{+}$; gray) T cells in brains from mice inoculated either i.n. $(n=5)$ or i.v. $(n=3)$ with HK-CAS or from naive controls $(n=1)$. Data represent the mean \pm SEM. (D) FACS plots of CD44 hiCD4+ $T$ cells isolated from brains of either naive or HK-CAS-inoculated mice that were activated for 3 hours ex vivo with PMA+I prior to IL-17A and IFN- $\gamma$ analysis. Gray arrow shows the percentage of bacterium-specific (2W:I-A $\left.{ }^{b+}\right)$ T cells that expressed either IL-17A or IFN- $\gamma$.

marily ( 60\%) localized in the rostral brain regions (bregma 4.28-3.0), encompassing the olfactory bulb (OB) and anterior olfactory nucleus (AON) (Figure 2, D, F, and K; Supplemental Figure 2, B-D; and Supplemental Figure 4D). We observed very few $\mathrm{T}$ cells in tissues from either singly infected or naive mice, as previously reported (25) (Figure 2, C, E, and K; Supplemental Figure 2A; and Supplemental Figure 4, A and C). Most CD4 ${ }^{+} \mathrm{T}$ cells in GASimmune mice were associated with the olfactory nerve $(75 \%)$ and glomerular layers $(15 \%)$, where olfactory sensory axons enter the brain and form synapses with the dendrites of mitral and tufted cells (26) (Figure 2D, and Supplemental Figure 2, B and C). Rarely, we found that $\mathrm{CD} 4^{+} \mathrm{T}$ cells were located in close proximity to blood vessels (Figure $2 \mathrm{~F}$ ), suggesting that some may have entered from the vasculature. $\mathrm{CD} 4^{+} \mathrm{T}$ cells in more caudal brain regions (bregma 3.0 to -2.46 ) were primarily found in areas where OB output neurons (mitral and tufted cells) make synaptic connections with areas such as the AON, the olfactory tubercle (OT), the piriform cortex (PC), the amygdala, the lateral hypothalamus ( $\mathrm{LH})$, as well as the basal ganglia; however, $\mathrm{T}$ cells were not seen in sections from similar regions of the brains from naive mice (Figure 2, G-K, and data not shown). Distribution of $\mathrm{CD} 4^{+} \mathrm{T}$ cells in the brains of GAS-infected SJL/J mice, a mouse strain previously shown to exhibit behavioral changes upon inoculation with GAS hyperimmune serum $(13,14)$, was similar to that seen in C57BL/6 mice 6 hours after the final inoculation (Figure $2 \mathrm{~K}$ and Supplemental Figure 2D). However, we found that $\mathrm{CD} 4^{+} \mathrm{T}$ cells were distributed to more posterior brain regions (bregma -3.5 to 2.5) 48 hours after the final inoculation (Supplemental Figure 2D). These $\mathrm{T}$ cells had either migrated from more anterior CNS compartments or crossed a leaky BBB. The distribution and extensive colocalization of $\mathrm{CD} 4^{+}$ $\mathrm{T}$ cells with olfactory sensory axons en route to the OB (Figure 2D and Supplemental Figure 2B) suggests that $\mathrm{T}$ cells most likely enter the $\mathrm{OB}$ via the cribriform plate and travel along olfactory sensory axons before they disperse into more caudal brain regions.

Since virus-specific $\mathrm{T}$ cells remain in the brains of mice for at least 150 days (11), we questioned whether streptococcus-specific $\mathrm{T}$ cells also persist in the brain over time. $2 \mathrm{~W}: \mathrm{I}-\mathrm{A}^{\mathrm{b}+} \mathrm{T}$ cells were not detected in the brains of naive mice or in those of mice that had received a single i.n. GAS inoculation; however, these cells persisted in the brains of mice immunized with multiple GAS inoculations for at least 56 days after the last inoculation (Figure 2L).

Although the mice were perfused with PBS prior to brain isolation, it is possible that some of the recovered T cells did not reside 
A

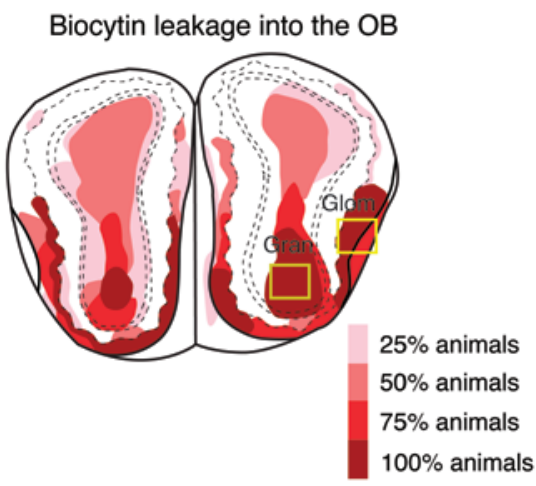

$\mathbf{F}$

Biocytin leakage into the posterior brain

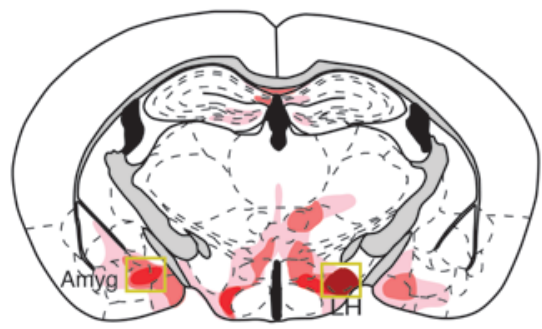

Biocytin-TMR Caveolin 1
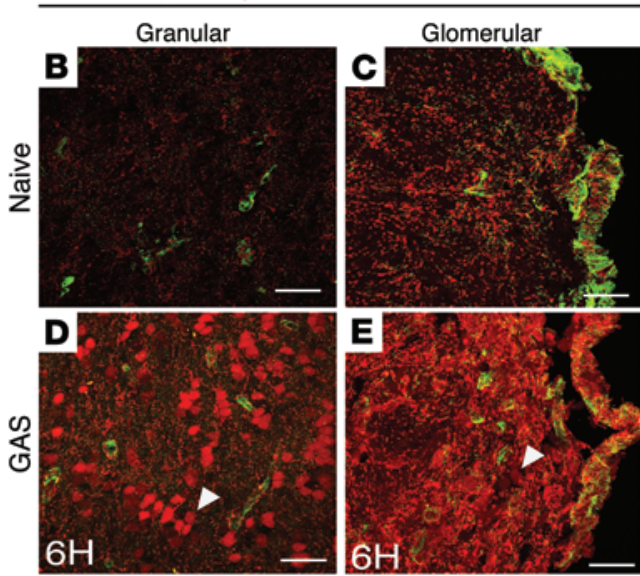

Biocytin-TMR Caveolin 1
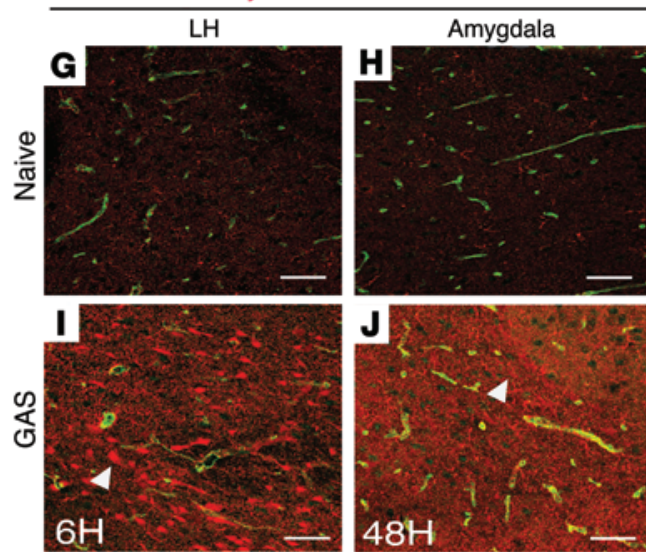
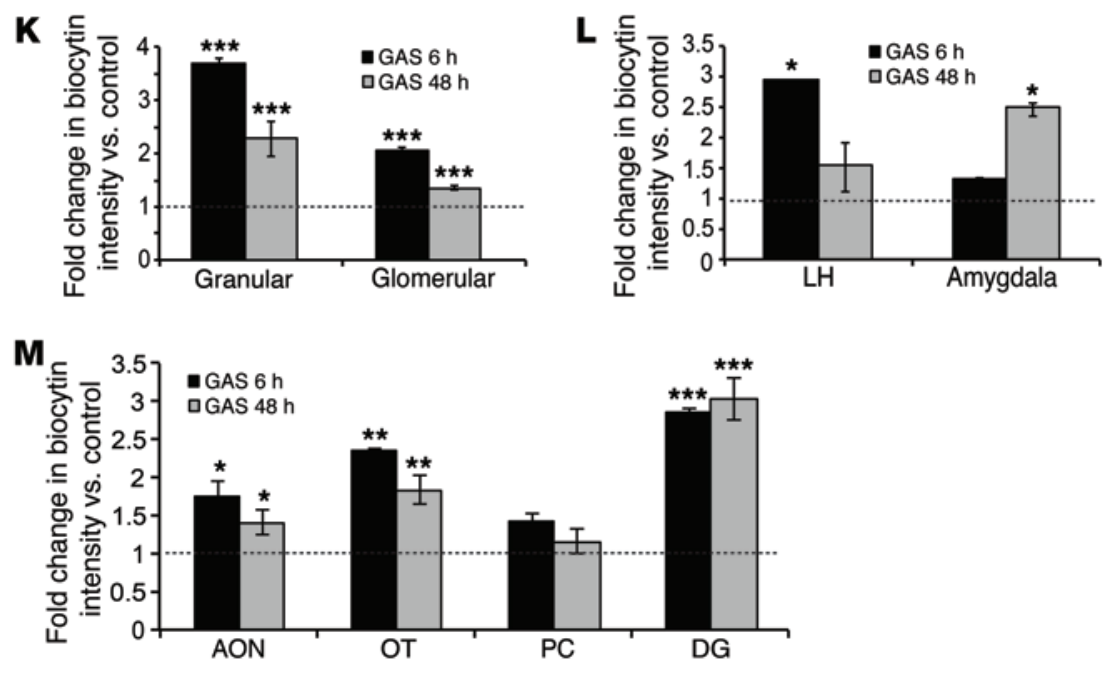

in the brain parenchyma but rather in luminal or abluminal vascular spaces. Therefore, we determined the fraction of luminal versus abluminal vascular and/or parenchymal $\mathrm{T}$ cells in the brain by administering CD90.2 Ab to multiply GAS-infected mice prior to isolating $\mathrm{T}$ cells from the brain. Intravenously injected CD90.2 $\mathrm{Ab}$ is known to bind to the surface of luminal, but not abluminal, vascular or parenchymal T cells (27). We found that the majority of $\mathrm{CD} 44^{\mathrm{hi}} \mathrm{CD} 4^{+} \mathrm{T}$ cells $(93 \%)$ from brains did not bind the antimouse CD90.2 Ab (Supplemental Figure 2E), indicating that these
T cells resided either in the abluminal vascular space and/or brain parenchyma and not in the luminal vascular space. Therefore, perfusion with buffer eliminated most, but not all, luminal vascular $\mathrm{T}$ cells from the brain, which is consistent with the presence of few $\mathrm{CD} 4^{+} \mathrm{T}$ cells in the brains of naive animals (Figure 2B).

GAS-specific Th17 cells in the brain likely originate from NALT. The cytokine profiles of $\mathrm{T}$ cells from the brains of mice that had multiple inoculations were investigated after the last i.n. exposure to live streptococci. We observed that IL-17A+ GAS-specific CD4 ${ }^{+}$ 
A

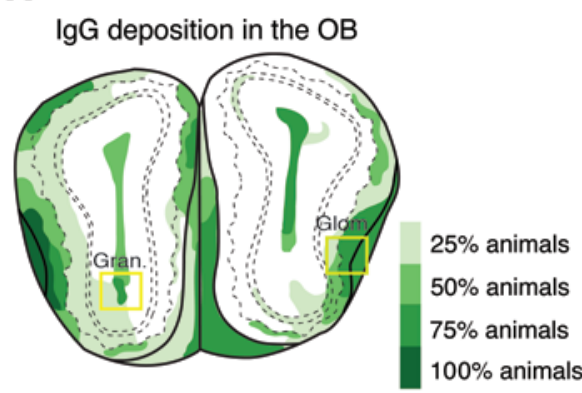

F IgG deposition in the posterior brain

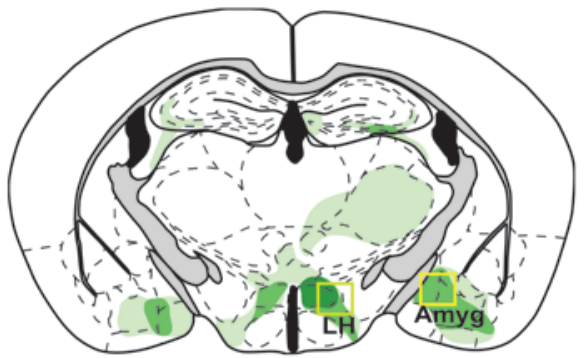

Glut1 Mouse IgG
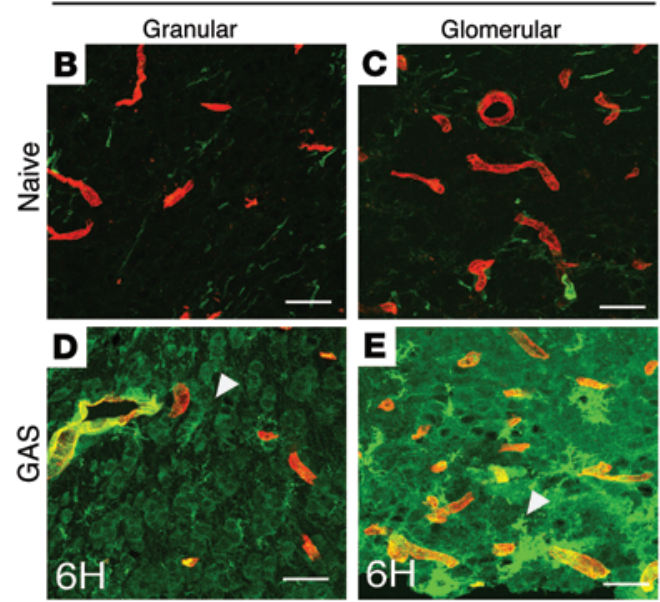

Glut1 Mouse IgG
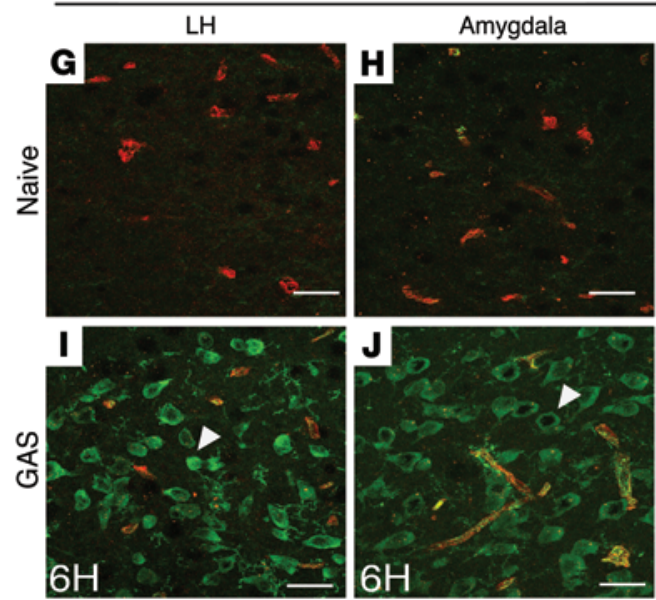

Figure 5 . T cell infiltration is accompanied by IgC deposition in the brain. ( $\mathbf{A}$ and $\mathbf{F}$ ) Heatmaps of IgG leakage distribution in the $\mathrm{OB}(\mathbf{A})$ and posterior brain $(\mathbf{F})$ in multiply infected SJL/J mice 6 hours and 48 hours after the last inoculation, respectively. Green hues represent the percentage of animals showing IgC in the brain, not the amount of IgC leakage among animals (see legend in $\mathbf{A}$ ). Yellow boxes in $\mathbf{A}$ and $\mathbf{D}$ outline the regions shown in B-E and G-J. Representative images of lgG leakage from blood vessels into granular and glomerular layers of the OB (B-E), LH (G and I), and amygdala ( $\mathbf{H}$ and $\mathbf{J}$ ) in CAS-inoculated and control naive mice. IgG extravasation occurred only in CAS-inoculated mice. (K-M) Bar graphs of the fold change in IgC average intensity of GAS-inoculated versus naive mice (dashed black line) in either the $\mathrm{OB}$ (K) or LH and amygdala (L), or in other brain regions $(M)$ (AON,OT, $P C$, and DC) 6 hours (black bars) and 48 hours (gray bars) after the last inoculation. Baseline fluorescence intensity in control naive animals is indicated by a dashed black line. Data were collected from 3 independent experiments for 3 to 4 animals and are presented as the mean \pm SEM. ${ }^{*} P<0.05$, by 2 -tailed Student's $t$ test comparison between CAS-inoculated and naive animals. Scale bars: $50 \mu \mathrm{m}$ (B-E and G-J).
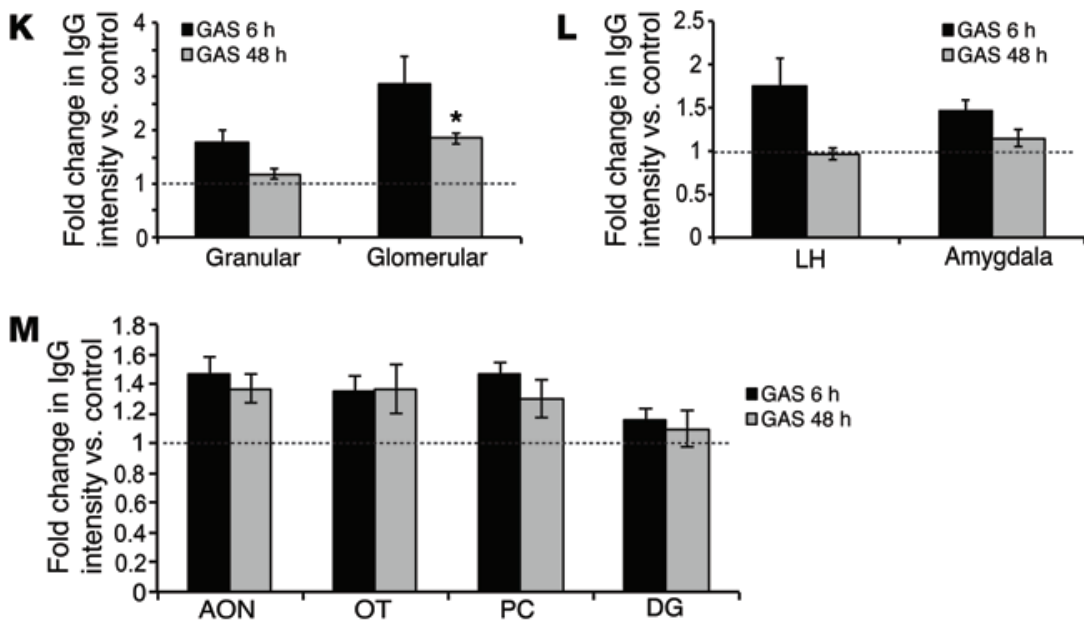

T cells dominated $\mathrm{T}$ cell populations in brains, NALT, and CLNs (Figure 3A). Most GAS-specific T cells (up to 90\%) isolated from brains expressed IL-17A, and approximately 50\% expressed both IL-17A and IFN- $\gamma$ (Figure 3, A and B). We found similar cytokine profiles for GAS-specific CD4 ${ }^{+} \mathrm{T}$ cells isolated from NALT of the same mice. IL-17A+IFN- $\gamma^{+}$T cells were not common in CLNs, but they dominated IL-17A ${ }^{+} \mathrm{T}$ cell populations in both brains and NALT (Figure 3, A and B).
The similarity in cytokine expression profiles of $\mathrm{T}$ cells from brain and NALT (Figure 3B) suggests that blood was not the primary source of the $\mathrm{T}$ cells found in the brain. Consistent with this finding, i.v. vaccination of mice with HK-GAS, a route known to generate primarily Th1 cellular responses (17), did not result in $\mathrm{T}$ cell migration to the brain, contrary to what we observed with i.n. HK-GAS vaccination, which resulted in extensive GAS-2Wspecific $\mathrm{T}$ cell accumulation in the brain (Figure 3, C and D, and 

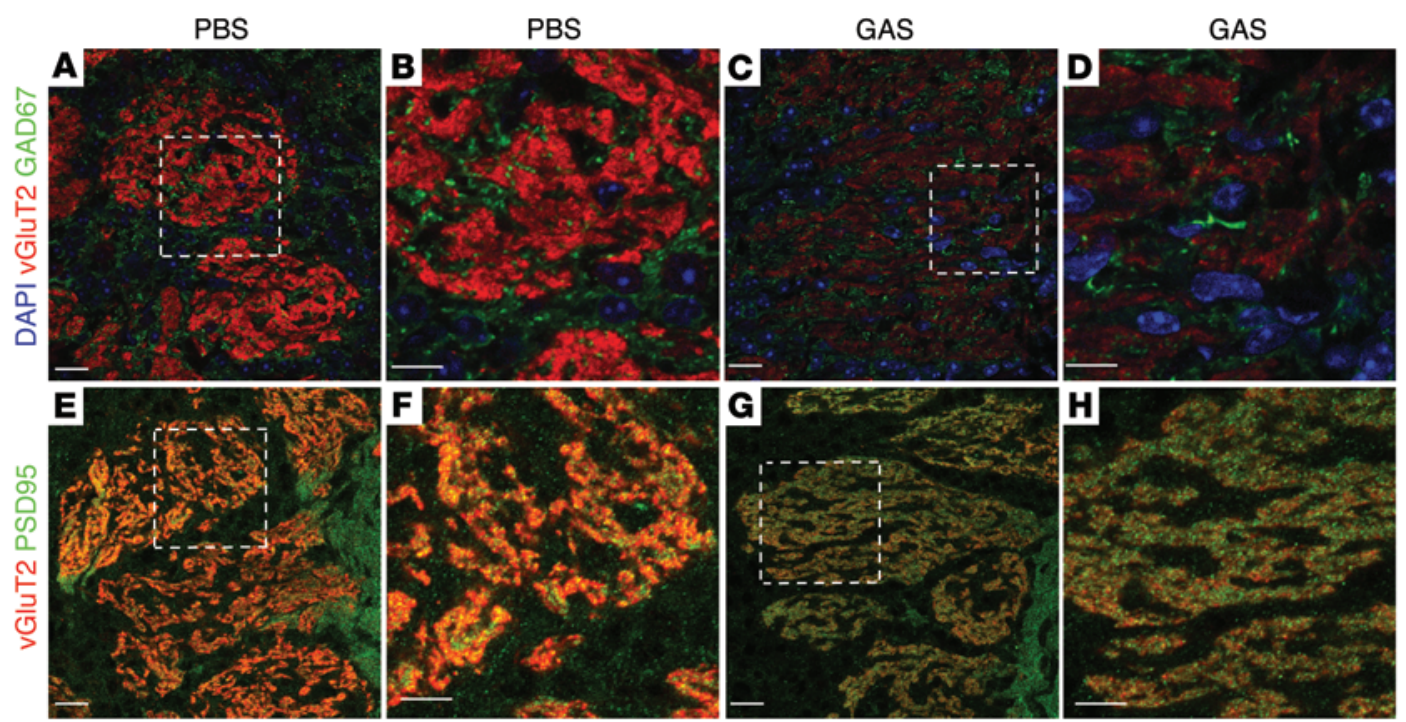

1

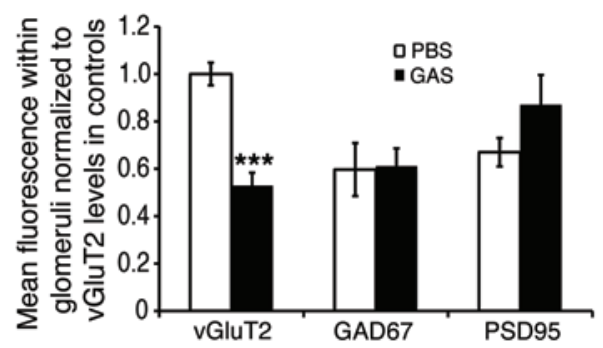

$\mathbf{J}$

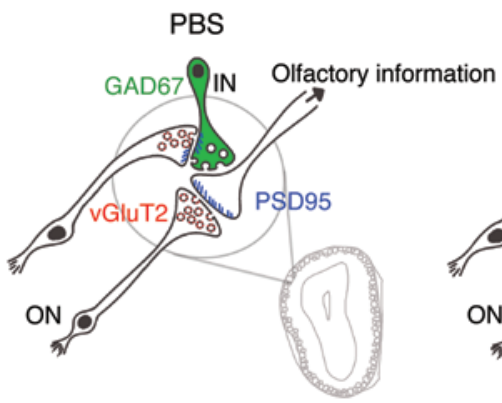

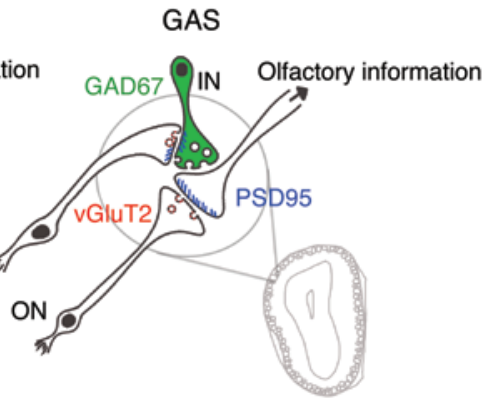

Figure 6. $T$ cell accumulation in the $\mathrm{OB}$ is accompanied by loss of vesicular excitatory synaptic proteins. (A-H) Immunofluorescence images of olfactory glomeruli stained for the vesicular marker vGluT2 (red) and GAD67 (green, A-D) or PSD95 (green, E-H). Scale bars: $15 \mu \mathrm{m}$ (A, C, E, and G) and $5 \mu \mathrm{m}$ (B, D, $\mathbf{F}$, and $\mathbf{H}$ ). (I) Bar graphs of fluorescence intensity for vGluT2, GAD67, and PSD95 within glomeruli. Data were collected from 3 to 5 mice in 2 independent experiments and represent the mean $\pm \mathrm{SEM}$. ${ }^{* *} P \leq 0.001$, by 1-way ANOVA with Tukey's post-hoc multiple comparisons test. (J) Simplified diagram of the synaptic circuitry within olfactory glomeruli, which receive excitatory input from the nose via vGluT2-expressing olfactory sensory neurons (ON). Olfactory information is modulated by inhibitory interneurons (IN) that express GAD67 before it is relayed to posterior parts of the brain, including limbic regions. PSD95 (blue) is expressed in the postsynaptic component of mitral or tufted cell dendrites within the olfactory glomeruli that make connections with olfactory sensory axons. The number of vGluT2 vesicles in the presynaptic component (olfactory sensory axons) is reduced after repeated i.n. GAS infections.

Supplemental Figure 3, A, E, H, K, and N). Moreover, NALTderived IL-17A ${ }^{+}$T cells purified from GAS-immunized mice did not home to the brain after adoptive transfer into naive animals via tail vein injection (data not shown), suggesting that both the Th17 phenotype of GAS-specific T cells and T cell activation in NALT are important for subsequent $\mathrm{T}$ cell migration to the brain.

In order to evaluate whether induction and passage of GASspecific Th17 cells into the brain is specific for streptococci and not due to a nonspecific inflammatory response to olfactory mucosal infection per se, infections were repeated with two different heat-killed bacterial pathogens that express the same $2 \mathrm{~W}$ epitope, Listeria monocytogenes-2W (LM-2W) (28) and Salmonella typhimurium-2W (ST-2W) (29). Listeria is Gram positive and, like GAS, produces pathogen-associated molecular patterns (PAMPs) such as lipoteichoic acid (LTA), whereas Gram-negative Salmonella produces LPS, another highly active PAMP. Listeria and Salmonella induce robust Th1 cellular responses following systemic infections in mice and weak Th17 responses after i.n. inoculations. Heat-killed bacteria were used because mice do not survive live S. typhimurium infections. As expected, multiple i.n. inoculations with HK-GAS-2W resulted in more than $4 \times 10^{3} \mathrm{CD} 4^{+} \mathrm{CD} 44^{\mathrm{hi}}$ and several hundred GAS-specific $2 \mathrm{~W}: \mathrm{I}-\mathrm{A}^{\mathrm{b}+} \mathrm{T}$ cells in the brains of mice (Supplemental Figure 3A). Contrary to what we observed with GAS-2W, inoculations with $L M-2 \mathrm{~W}$ or $S T-2 \mathrm{~W}$ resulted in only small numbers of $\mathrm{CD} 4^{+} \mathrm{CD} 44^{\mathrm{hi}} \mathrm{T}$ cells and no bacteriumspecific $2 \mathrm{~W}: \mathrm{I}^{\mathrm{b}} \mathrm{A}^{\mathrm{b}} \mathrm{T}$ cells in mouse brains (Supplemental Figure $3 \mathrm{~A})$. Although mice were inoculated with equal numbers of each bacterial species, the bacterium-specific (2W:I-Ab+) CD4 T cell expansion and $\mathrm{IL}^{-17 \mathrm{~A}^{+}}$cellular response to LM-2W and ST-2W in NALT were significantly weaker than the expansion and response to GAS-2W (Supplemental Figure 3, B and C). To ensure both the expression and presence of the $2 \mathrm{~W}$ epitope in the heatkilled preparations of $L M-2 \mathrm{~W}$ and $S T-2 \mathrm{~W}$, mice were inoculated i.v. with either HK-GAS-2W, HK-LM-2W, or HK-ST-2W from the same preparations that were used for the i.n. inoculations. One week after the primary i.v. inoculation, spleens were harvested and analyzed for $2 \mathrm{~W}: \mathrm{I}^{-A^{b+}} \mathrm{CD} 4^{+} \mathrm{T}$ cells. Few naive $\mathrm{CD} 4^{+} 2 \mathrm{~W}: \mathrm{I}^{-\mathrm{A}^{\mathrm{b}}}$ $\mathrm{CD} 44^{10}$ cells present in the naive mice expanded and upregulated CD44 in response to the i.v. inoculations of heat-killed bacterial preparations, indicating the presence and proper processing of $2 \mathrm{~W}$ 

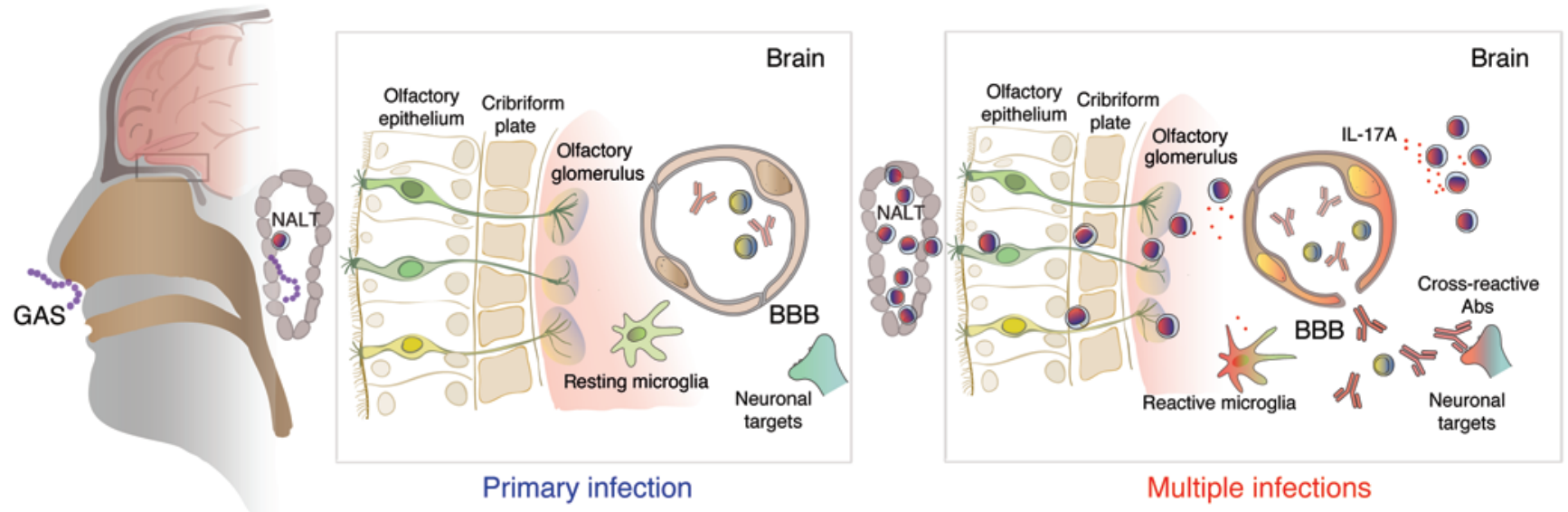

Figure 7. GAS-specific Th17 cells migrate into the brain through the cribriform plate along olfactory sensory axons and induce BBB breakdown and lgG extravasation. Our model illustrates two scenarios for primary and multiple mucosal GAS exposures. Multiple GAS exposures predominantly expand Th17 T cells. These effector memory T cells occupy the NALT, including Waldeyer's ring and other lymphoid tissues that decorate the nasal mucosa in the mouse (the adenoids and palatine tonsils are the equivalent human lymphoid tissue in proximity to nasal mucosa). The association of CD4+ $T$ cells (spheres with red-blue centers) with olfactory sensory axons in glomeruli suggests that axons provide a potential route for T cell migration from the olfactory mucosa to the $\mathrm{OB}$, from where they spread into other CNS regions. We postulate that IL-17A and other cytokines produced by these T cells activate microglia and induce BBB leakage, resulting in extravasation of Abs and additional circulating T cells into the brain. Autoantibodies induced by GAS would then target mimetic brain antigens (e.g., dopamine receptors) to induce abnormal motor and/or behavioral changes.

epitope in HK-LM-2W and HK-ST-2W (Supplemental Figure 3D). In a separate experiment, mouse brains were sectioned, stained, and examined by immunofluorescence for the presence of $\mathrm{CD} 4^{+}$ $\mathrm{T}$ cells. We found $\mathrm{T}$ cells in the brains of mice from each of the three inoculation groups (Supplemental Figure 3, E-N); however, the number and distribution of these $\mathrm{T}$ cells differed among the species. GAS-inoculated animals had significantly more $\mathrm{T}$ cells in their OBs and brains than did mice inoculated with S. typhimirium or L. monocytogenes (Supplemental Figure $3 \mathrm{~N}$ ). The low number of $\mathrm{T}$ cells in the choroid plexus was similar in all infected mice, regardless of the pathogen (Supplemental Figure 3, K, L, and M). These findings indicate that only i.n. GAS infections elicit a unique and robust cellular immune response that produces $\mathrm{T}$ cells capable of traveling to the brain.

Migration of $T$ cells to the brain does not require infection per se. An important question is whether the observed $\mathrm{T}$ cell homing to the brain required tissue infection. Our previous observations that infection-immunized mice clear streptococci from the NALT within 24 hours, rather than 7 days compared with naive mice (17, 18), suggested that actual infection of the brain is not required for the recruitment of $\mathrm{T}$ cells to the CNS. As shown above, multiple i.n. inoculations with HK-GAS-2W induced CD $44^{\text {hi }} 2 \mathrm{~W}: \mathrm{I}^{-\mathrm{A}^{\mathrm{b}}} \mathrm{CD}^{+}$ IL- $17 A^{+} T$ cells to home to the brain, albeit in smaller numbers than was observed with live bacteria (Figure 3, C and D, and Supplemental Figure 3, A and N). In addition, single-cell brain suspensions from mice infected i.n. with live streptococci failed to yield viable bacterial colonies on blood agar plates (data not shown). Finally, we did not detect GAS in sections of $\mathrm{OB}$ or other brain regions from infected mice stained with an anti-group A carbohydrate-specific $\mathrm{Ab}$, although bacteria were very abundant along the nasal olfactory epithelium (Supplemental Figure 4, A-D, and data not shown). On the contrary, we observed many $\mathrm{CD} 4^{+} \mathrm{T}$ cells in both the nasal epithelium and OBs from inoculated mice, but not from naive mice
(Supplemental Figure 4, A-D). These observations suggest that immunized mice block the dissemination of streptococci from the olfactory epithelium and that streptococci are not required to reside in the brain in order to trigger infiltration of Th17 cells.

The presence of $C D 4^{+} T$ cells in the brain is associated with $B B B$ leakage, disruption of endothelial tight junctions, and deposition of serum IgG. We analyzed the neurovascular integrity of the BBB in GAS-immunized mice by mapping the leakage of a low molecularweight (MW) fluorescent tracer, 5-(and-6)-tetramethylrhodamine biocytin (biocytin-TMR, MW $=869 \mathrm{Da}$ ). Biocytin-TMR crosses the BBB when paracellular, but not transcellular, barrier permeability is enhanced (30). The tracer was introduced into the tail vein of multiply GAS-inoculated mice either 6 or 48 hours after the final inoculation or into the tail vein of naive control mice. We assessed the amount of biocytin-TMR leakage by comparing fold increases in the average fluorescence pixel intensity of the tracer present in brain sections from GAS-inoculated mice and naive controls (brain regions such as the choroid plexus, the circumventricular organs around the third ventricle, and the area postrema, which normally have a leaky vasculature, were excluded from this analysis). Biocytin-TMR was consistently present at significantly higher levels in the OB (2- to 3.5-fold higher levels in GAS-inoculated mice than in naive mice; $P<0.05)$. In $100 \%$ of the mice analyzed, we found that tracer leakage was most pronounced in the glomerular and granular layers (Figure 4, A-E and K, and Supplemental Figure 6, K and L), where large numbers of $\mathrm{CD} 4^{+}$cells were also localized (Figure 2, D, F, and K, and Supplemental Figure 4D). Moreover, biocytin-TMR was also present in brain regions such as the AON, OT, LH, and amygdala, where OB projection neurons make synaptic connections (Figure $4, \mathrm{~F}-\mathrm{J}, \mathrm{L}$, and $\mathrm{M}$ ). The extent of BBB leakage within the $\mathrm{LH}$ and amygdala was somewhat variable. Blood vessels in the LH showed leakage 6 hours, but not 48 hours, after the final GAS inoculation (Figure 4, I, J, and L), whereas leak- 
age was most pronounced in the amygdala 48 hours after the final inoculation (Figure 4, I, J, and L). The delayed increase in BBB permeability in the amygdala may reflect a release of catecholamines into the bloodstream that can selectively upregulate regional paracellular BBB permeability (31). We found that BBB impairment in the basal ganglia and piriform cortex was less consistent and correlated with fewer $\mathrm{CD} 4^{+} \mathrm{T}$ cells in those regions (Figure 2, H, J, and $\mathrm{K}$ ). Moreover, minor BBB leakage was inconsistently found in the glomerular and granular layers of the OB 6 hours after a single i.n. GAS infection (Supplemental Figure 5, A, B, and E). Therefore, multiple i.n. GAS inoculations that amplify streptococcus-specific IL $-17^{+} \mathrm{T}$ cells also impair BBB function.

Previous studies showed that i.v. injection into mice of serum from animals immunized with HK-GAS (13) or s.c. immunization of rats with crude protein extracts emulsified in CFA (14) promoted the deposition of GAS-specific Abs into the brains of recipient rodents and induced behavioral deficits. Simultaneous administration of LPS or pertussis toxin was required for these changes in both models. We postulated that serum IgG would extravasate into brain areas where the $\mathrm{BBB}$ was damaged in our animal model; therefore, brain sections from naive mice or singly or multiply GAS-inoculated mice were stained with anti-mouse IgG. The amount of IgG was estimated by the fold increase in pixel intensity relative to that in naive mice (30). We observed extensive (1.5- to 3.0-fold increase; $\left.{ }^{*} P<0.05\right)$ IgG leakage within granular and glomerular layers of the OB from GAS-inoculated mice (Figure 5, A, D, E, and K, and Supplemental Figure 6N) compared with that seen in control mice (Figure 5, A-E and K, and Supplemental Figure $6 \mathrm{~N}$ ); these areas also displayed extensive biocytin-TMR leakage. Moreover, IgG deposition was inconsistently found in the LH or amygdala in multiply GAS-infected mice (Figure 5, F-J and L) compared with uninfected controls (Figure 5, G, H, and L). Although IgG deposition was present in other brain regions (e.g., AON, OT, PC, and dentate gyrus [DG]) of GAS-inoculated mice, there were no significant differences in IgG fluorescence intensities between GAS-inoculated and naive animals (Figure 5M). Minor biocytin-TMR leakage was observed in the glomerular and granular regions of the OB 48 hours after a single i.n. challenge (Supplemental Figure 5, A-F). However, the amount of IgG deposition and number of T cells (data not shown) in OB sections from singly inoculated animals were not different from those detected in controls (Supplemental Figure 5G). Moreover, no significant differences in biocytin-TMR leakage, IgG deposits, or T cells were observed in other regions of the brains of singly inoculated mice compared with brains of naive animals (Supplemental Figure 5, F and $\mathrm{H}$, and data not shown).

IL-17A is known to disrupt endothelial tight junctions (TJs) in both human MS and mouse models of the disease (21). We therefore investigated whether enhanced paracellular BBB permeability correlated with disruption of BBB TJs. Expression and subcellular localization of the endothelial TJ proteins claudin- 5 and ZO-1 in blood vessels of OBs from naive or GAS-inoculated mice were analyzed for additional evidence of profound BBB breakdown. We found that endothelial TJs had extensive gaps and protrusions, two structural abnormalities present during TJ remodeling (30) in multiply, but not singly, GAS-inoculated mice or uninfected controls (Supplemental Figure 6, A-J). A significant fraction of claudin-5- and ZO-1-positive TJ strands had gaps ( 35\%) and protrusions ( $25 \%)$, suggesting that TJs were undergoing structural remodeling within the brain vasculature (Supplemental Figure 6, A-J). These findings suggest that GAS-specific Th17 cells enter the brain and express IL-17A, which induces endothelial TJ breakdown, leading to increased $\mathrm{BBB}$ paracellular permeability and extravasation of IgG from the blood into the brain.

Repeated i.n. GAS inoculations result in microglial activation and loss of excitatory synaptic protein to the $O B$. We anticipated that GAS-specific Th17 cells in the brain would be accompanied by inflammation and activation of microglia, CNS-resident immune cells that are known to respond to T cell-derived cytokines (32, 33). Therefore, we analyzed the activation state of microglia in the brains of multiply GAS-inoculated mice. The number of $\mathrm{CD} 8^{+} \mathrm{Iba}^{+}$activated microglia in the glomerular layer of OB sections from multiply GAS-inoculated mice was 3-fold greater than in tissue from uninfected controls (Supplemental Figure 7, A-F, and J). We observed that the majority of activated microglia were localized within glomeruli in close proximity to $\mathrm{CD} 4^{+} \mathrm{T}$ cells (Supplemental Figure 7, G-I). Therefore, i.n. exposure of infectionimmunized mice to GAS caused $\mathrm{CD}^{+} \mathrm{T}$ cell migration into the $\mathrm{OB}$, with local activation of microglia and no evidence of viable bacteria in the tissue (Supplemental Figure 4D).

Inflammation and microglial activation are known to result in synapse elimination (34-36). Olfactory neurons that project excitatory information into the $\mathrm{OB}$ may be particularly vulnerable to inflammatory injury, since their cell bodies reside within the mucosal epithelial layers of the nose. The excitatory presynaptic marker vGluT2 is highly expressed in terminals of olfactory sensory axons that establish synapses with second-order neurons (mitral and tufted cells) in the glomerular layer (37) and colocalizes with the postsynaptic marker PSD95. The GABA transporter protein GAD67 is interspersed throughout glomeruli in a subset of local interneurons that modulate excitatory information from the nose to the brain (Figure 6J). While it is not possible to visualize individual synapses in the glomerular layer of the OB due to high synaptic density, we evaluated the effect of multiple GAS infections on synaptic processing by monitoring the expression of primary synaptic proteins using immunofluorescence microscopy (Figure 6, A-H). We found that the levels of vGluT2 within glomeruli were significantly reduced after multiple i.n. GAS inoculations, whereas the levels of the inhibitory interneuron marker GAD67 were unchanged (Figure 6, C, D, and I), suggesting a change in signal processing within the OB. To determine whether the decrease in excitatory input was due to loss of presynaptic input only or to loss of the complete synapse, levels of the postsynaptic marker PSD95 were also analyzed (Figure 6, E-H). We observed no significant differences in PSD95 levels within the glomeruli between naive and multiply GAS-inoculated mice, suggesting that the postsynaptic components were intact (Figure 6, E-I). We hypothesize that multiple GAS inoculations reduce excitatory signals projecting to the $\mathrm{OB}$. This aberrant information is then transmitted to more caudal brain regions and may underlie some component of the neuropathology (Figure 6J).

\section{Discussion}

The pathophysiological triggers of initial onset and subsequent sporadic exacerbations of movement and neuropsychiatric dis- 
orders associated with several autoimmune-mediated CNS diseases, including those linked to GAS infections, are not understood. Although autoantibodies that recognize neurotransmitter receptors, signaling kinases, and/or ion channels are thought to be directly responsible for symptoms $(8,38,39)$, the mechanisms by which autoantibodies cross the BBB remain unclear. We previously demonstrated that Th17 cells are the dominant response to multiple GAS i.n. infections in the mouse model used here $(17,18)$. The link between Th17 cells and several autoimmune conditions, including MS (12), prompted us to question whether GAS-specific $\mathrm{T}$ cells home to the brain.

Using peptide-MHCII tetramers to concentrate and identify antigen-specific $\mathrm{T}$ cells, we show that GAS-specific Th17 cells generated in the NALT migrate into the OB, the first relay station where olfactory information is processed, and then disperse to other brain regions. Colocalization with olfactory sensory axons suggests that $\mathrm{T}$ cells travel along the olfactory sensory pathway. GAS-specific Th17 cell entry into the brain is associated with impairment in both structural and functional BBB properties that in turn permit circulating IgG and additional CD $4^{+} \mathrm{T}$ cells to enter the brain, presumably recognize their cognate neuronal targets, promote neuroinflammation (e.g., activate microglia), and alter synaptic connectivity. Administration of LPS or pertussis toxin to mice or rats either passively or actively immunized with GAS results in both IgG deposition in the brain and behavioral changes $(13,14)$; however, our data are the first to our knowledge to suggest a mechanism by which GAS infections opens a window for autoantibodies to access CNS targets.

Why do T cells home to and persist in the brain? It is unclear why GAS-specific T cells migrate to the brain. Recruitment of GASspecific $\mathrm{T}$ cells to the brain and activation of IL-17A do not appear to require the physical presence of viable streptococci in the CNS. The immune response induced by three or more i.n. inoculations, as described here, clears viable streptococci from NALT within 24 hours, and mice appear physically healthy $(17,18)$. Yet 6 hours after the last inoculation, large numbers of GAS were observed along the olfactory mucosa, while streptococci were not detected in the $\mathrm{OB}$ or other brain regions (Supplemental Figure 4, A-D, and data not shown). We presume that failure to observe streptococci in the $\mathrm{OB}$ of GAS-inoculated mice indicates that mucosal immunity prevents dissemination of bacteria into the brain. The presence of GAS$2 \mathrm{~W}$-specific $\mathrm{T}$ cells in the brains of mice that lack the $2 \mathrm{~W}$ epitope indicates that autoreactivity is not a prerequisite for their migration to and persistence in that organ. However, brains from multiply infected, but not naive, mice also contained significant numbers of $\mathrm{CD}^{+} \mathrm{T}$ cells with unknown specificities, so it is conceivable that some of these could be autoreactive T cells. Whether GAS-specific $\mathrm{T}$ cells passively move into the brain or are actively recruited by resident antigen-processing macrophages, microglia, or olfactory ensheathing glia, all of which are known to phagocytize bacterial debris (40), is an important unanswered question. Once in the brain, Th17 cells likely persist due to high levels of CCR6 and LFA-1 proteins, both of which have previously been shown to be required for $\mathrm{CD}^{+} \mathrm{T}$ cells to remain in the brains of mice with experimental autoimmune encephalomyelitis (EAE) (41).

It is also unclear how GAS-specific T cells are activated to produce IL-17A within 6 hours of introducing HK-GAS into the nares of immune mice, a week or more after the previous inoculation. Accordingly, our experiments could not distinguish between activation of CNS-resident $\mathrm{T}$ cells or activation and trafficking of new $\mathrm{T}$ cell immigrants from the NALT. $\mathrm{CD}^{+} \mathrm{T}$ cells can persist in mouse brains for over 150 days; however, reactivation of those cells required reintroduction of virus into the brain (11). Antigenpresenting cells from the NALT may migrate into the brain and present cognate peptides to preexisting GAS-specific $\mathrm{T}$ cells. Alternatively, antigens may be transported by phagocytic olfactory ensheathing glia (42). Streptococcus pneumoniae and bacterial toxins interact with asialo-GM2 gangliosides on neuronal surfaces and travel retrogradely along olfactory sensory axons (43). Thus, GAS antigens could also travel along sensory axons to the $\mathrm{OB}$ and be presented to GAS-specific Th17 cells by local microglia.

GAS-specific Th17 cells travel from the NALT via an olfactory route to the brain. Although we do not have direct evidence, our data are best explained by proposing that GAS-specific Th17 cells migrate from the NALT through the cribriform plate along olfactory sensory axons in order reach the brain. Several lines of evidence from our study support this conclusion: (a) T cells were located predominantly along the outer nerve layer of the $\mathrm{OB}$, a region with extensive $\mathrm{BBB}$ leakage and IgG deposition, just 6 hours after i.n. exposure of immune mice to either live or HK-GAS; (b) cytokine profiles of $\mathrm{T}$ cells isolated from the brain were similar to those from NALT and different from $\mathrm{T}$ cells obtained from CLNs and blood; (c) i.v. immunization and challenge with HK-GAS did not promote $\mathrm{T}$ cell migration into the brain; (d) i.n. infections with heat-killed Salmonella or Listeria that generally produce weak Th17 responses, did not result in significant $\mathrm{T}$ cell migration to the brain; and (e) i.v. transfer of Th17 cells harvested from NALTs of multiply GAS-inoculated mice did not result in T cell translocation into the brains of naive mice in the absence of i.n. exposure to streptococci (data not shown). Therefore, migration of T cells into the brain requires substantial expansion of effector memory Th17 cells in close proximity to the olfactory mucosa. CD $4^{+} \mathrm{T}$ cells can enter the CNS either via the choroid plexus (44), specific "gates" in white matter regions stimulated by regional neural activation (45), or through a recently discovered functional lymphatic system that links the brain to deep CLNs $(46,47)$, thus bypassing the intact BBB. However, these mechanisms are unlikely to have played a significant role in our mouse model, since they are inconsistent with the observed distribution of $\mathrm{T}$ cells in the brain.

Is BBB leakage induced by local olfactory mucosal inflammation, $I L-17 A^{+} T$ cells, or both? Many components of the immune response could induce regional BBB permeability in response to infection; however, given our findings, we argue that migrant, GAS-specific, activated effector Th17 cells and the associated neuroinflammation are the primary cause of $\mathrm{BBB}$ leakage and subsequent damage to excitatory synapses. While serum proteins such as IgG have been reported to diffuse at low levels into the OB and hypothalamus of healthy animals $(48,49)$, repeated GAS inoculations increased brain IgG levels over those in controls. In the brains of multiply GAS-inoculated animals, $\mathrm{T}$ cells accumulated in regions that had large increases in BBB permeability, suggesting that the large numbers of Th17 cells that enter the brain mediate regional BBB breakdown. It is possible that local mucosal inflammation accounted for the small amounts of biocytin-TMR leakage in the OB induced by 
the initial infection of naive mice. However, in contrast to brains from animals immunized by multiple i.n. GAS inoculations, we observed only minor leakage of biocytin-TMR, restricted to the OB region, in the brains of mice after a single i.n. GAS challenge. Moreover, we observed no significant increase in IgG deposits or T cells in the brains of these mice compared with those of naive mice, even though singly infected mice showed a significant inflammatory response, indicated by fever and other physical signs. We further postulate that in multiply GAS-infected mice, Th17 effector cells are activated in the NALT and then enter the OB through the cribriform plate. Once there, IL-17A and/or other cytokines produced by these cells could alter local BBB integrity, which allows additional $\mathrm{CD}^{+}{ }^{+} \mathrm{T}$ cells and circulating IgG to enter the brain parenchyma through the leaky vasculature. IL-17A disrupts TJs between endothelial cells in human MS and the EAE rodent model $(19,21)$, and Th17 cells were shown to form persistent synapse-like associations with neurons, resulting in loss of function (50). Furthermore, no significant increase in IgG deposits, disruption of endothelial TJs, activated microglia, or synaptic changes were observed in brains from naive mice after either a single or a second i.n. GAS challenge. Collectively, these results support our argument that BBB leakage is dependent on the infiltration of large numbers of GAS-specific Th17 cells into the brain and that nonspecific inflammatory responses are not sufficient to sustain BBB leakage and IgG deposition in the brain. We also hypothesize that after multiple immunizations by GAS infection, $\mathrm{T}$ cells retained in the OB from previous infections may be activated to produce inflammatory cytokines that disrupt the BBB following transport of GAS antigens into the brain from the olfactory mucosa. Future studies are required to elucidate the specific role of Th17 cells and their cytokines in the brain pathology after multiple GAS infections.

Consistent with the above hypotheses, the impaired BBB function observed in multiply GAS-inoculated mice coincides with abnormal gaps or protrusions in endothelial cell TJs, two structural defects that are also found during $\mathrm{TJ}$ remodeling in either epithelial cells under inflammatory conditions (51) or endothelial cells after ischemic stroke (30). Alternatively, recurrent GAS infections could upregulate expression of IgG Fc receptors on endothelial cells that promote the transport of serum IgG into the brain via receptor-mediated transcytosis (52). We cannot rule out the possibility that GAS-specific Abs themselves can open the BBB, since this has been reported for some other Ab types (53). However, the initial wave of $\mathrm{CD} 4^{+} \mathrm{T}$ cell migration into the $\mathrm{OB}$ may be sufficient to induce both local and more distal BBB damage, allowing additional $\mathrm{CD} 4^{+} \mathrm{T}$ cells and IgG to enter other brain regions, similar to the pathology described for EAE (12).

Our analysis of human tonsil tissue suggests that GAS-specific Th17 cells and significantly fewer Th1 cells populate the tonsils of children and adults. Incubation of cells with PMA+I confirmed the presence of large numbers of $\mathrm{CD} 4^{+} \mathrm{T}$ cells that are capable of expressing either IL-17A, IFN- $\gamma$, or both, as reported by Lim et al. (22). It is not possible to dismiss nonspecific activation of tonsilar $\mathrm{T}$ cells by GAS; however, the fact that Lactococcus bacteria (Figure 1D) did not induce T cells to produce IL-17A and/or IFN- $\gamma$ and that incubation with HK-GAS did not significantly activate IFN- $\gamma^{+}$T cells (expected from a nonspecific PAMP-TLR response) strongly argues for antigen specificity. Nevertheless, GAS and other bacteria produce superantigens and TLR ligands that could also nonspecifically activate IL-17A expression by Th17 cells and, in turn, disrupt the BBB and promote deposition of autoantibodies in the brain. Effector memory Th17 cells that populate the human mucosal and olfactory epithelium could also be a source of T cells for invasion of the human brain following multiple GAS infections. Several other bacterial or viral pathogens, including influenza virus, mycoplasma, and Staphylococcus aureus, induce robust Th17 responses (54-57) and therefore could also play a role in the exacerbation of behavioral symptoms in children with PANDAS if autoantibody levels are primed by previous GAS infections.

On the basis of our mouse data, we propose the following model to explain human motor and neuropsychiatric complications associated with multiple GAS infections (Figure 7). Although human palatine tonsils are anatomically located farther from the olfactory mucosa and cribriform plate than NALT in mice, adenoids and patches of lymphoid tissue that sporadically decorate nasal mucosae of children (58) are potential sources of GASspecific effector T cells. An initial upper respiratory GAS infection would induce the production of Abs that mimic and cross-react with CNS epitopes as well as prime a Th17 cellular response that may or may not be autoreactive. Subsequent GAS infections would further amplify both autoantibody levels and, in some individuals, increase the Th17 effector memory pool beyond a threshold required for cell migration from the mucosal epithelium into the $\mathrm{OB}$ along the olfactory nerve. Within the brain, the inflammatory response induced by IL-17A and/or activated microglia and macrophages then disrupts the $\mathrm{BBB}$, which permits autoreactive Abs to leak into the $\mathrm{OB}$ and other regions of the brain. The connection between GAS and behavioral exacerbations in PANDAS patients is confounded by occasional association with other non-streptococcal infections and the occasional failure to find evidence of GAS infections $(4,5,7)$. Although Listeria and Salmonella failed to drive $\mathrm{T}$ cells into the brain in our mouse model, the above discordance could be explained by vigorous cellular immune responses to sporadic infection by other mucosal pathogens known to induce dominant Th17 responses (e.g., S. aureus). Acute expansion of Th17 cells accompanied by a flood of IL-17A from NALT could acutely open the BBB to preexisting circulating autoantibodies.

A link to pathological behavior is suggested by our discovery of a decrease in excitatory vGluT2 levels in multiply GAS-inoculated mice. Presynaptic proteins (e.g., vGluT2) regulate quantal exocytosis of transmitter-filled vesicles, and their dysregulation or deficiency in various CNS regions such as the cortex, amygdala, or subiculum is known to cause neuropsychiatric dysfunction (anxiolytic phenotype and some behavioral correlates of schizophrenia) $(59,60)$. Therefore, the synaptic changes observed after multiple GAS inoculations could underlie some behavioral deficits seen in patients with SC or PANDAS. Moreover, BBB leakage and IgG deposition in our mouse experiments were primarily observed in those regions involved with the sense of smell (OB, AON, OT, and $\mathrm{PC})$, hunger (LH), and emotional learning, aggression, fear, and anxiety (all involving the amygdala), which may explain some of the behavioral characteristics of PANDAS patients $(5,6,10)$. However, we did not find extensive BBB damage or IgG deposition in the basal ganglia, a CNS region implicated in motor and other behavioral abnormalities exhibited by SC and PANDAS patients. 
The rarity of these CNS conditions in patients with known streptococcal infections suggests a more complicated picture; the nature of symptoms could also be influenced by a patient's immunogenetics and/or the specificity as well as the affinity and concentration of autoantibodies induced by infections. Moreover, rheumatic fever patients and those with neurological complications have elevated levels of Abs against a variety of GAS antigens, suggesting an imbalanced adaptive immune response to GAS infections (1). The environmental, microbial product, or genetic triggers of these sequelae are unknown. A genetic predisposition to rheumatic fever (61) and the existence of rheumatogenic strains of GAS (62) have been suggested, but definitive epidemiologic and laboratory evidence is lacking. The unique association of rheumatic fever, SC, and PANDAS with GAS infections may in part be due to the exceptionally intense TGF- $\beta 1$ response to mucosal infections, which in turn promotes an unusually robust Th17 cellular response $(17,18)$ and could at the same time suppress the expansion of Tregs. An abundant literature also suggests that expression of host mimetic proteins and vigorous $\mathrm{Ab}$ responses to those proteins are precursors to these rare complications of streptococcal infections.

Our results establish what we believe to be evidence of a novel crosstalk between the CNS and cellular immunity to infections, which may contribute to understanding the pathogenesis of many other CNS autoimmune diseases. Flare-ups associated with several chronic autoimmune conditions may be produced by the expansion of Th17 cells and the activation of cytokines induced by relatively common bacterial or viral infections. Aberrant cytokine expression could then disrupt the $\mathrm{BBB}$ to permit preexisting circulating autoantibodies to enter the brain, engage neural targets, and trigger the sudden onset of clinical symptoms.

\section{Methods}

Mice. Five- to six-week-old C57BL/6, C57BL/6J, or SJL/J female mice were purchased from the National Cancer Institute or The Jackson Laboratory and housed under pathogen-free conditions. Mice inoculated with GAS were housed in ABSL2+ facilities.

Analysis of GAS-specific T cells in human tonsils. Samples were collected from 26 pediatric (ages 3-10 years) and 2 adult (ages 20 and 26 years) patients who had undergone tonsillectomies for various conditions including sleep apnea, hyperplasia, and recurrent tonsillitis with or without symptoms associated with PANDAS. Tissue not required for pathological analysis was obtained within 3 hours of surgery from the surgical pathology departments of the University of Minnesota Fairview Hospital (Minneapolis, Minnesota, USA) and Georgetown University Hospital (Washington, DC, USA). Tissue samples from Georgetown University were cut into small pieces $(1 \mathrm{~cm} \times 1 \mathrm{~cm} \times 0.4$ $\mathrm{cm}$ ), placed in sterile cryovials with $1 \mathrm{ml} \mathrm{FBS}$ and $10 \%$ DMSO, slowly frozen at $-80^{\circ} \mathrm{C}$ overnight, and shipped on dry ice the following day. Frozen specimens contained $25 \%$ fewer $\mathrm{CD}^{+} \mathrm{T}$ cells that could be activated with PMA+I $(50 \mathrm{ng} / \mathrm{ml}$ and $500 \mathrm{ng} / \mathrm{ml}$ final concentrations, respectively) compared with cell numbers in fresh tissue. However, freezing did not change the $\mathrm{Th} 17^{+} / \mathrm{Th} 1^{+} \mathrm{T}$ cell ratio.

Fresh or thawed, previously frozen tissues were suspended in $5 \mathrm{ml}$ complete RPMI medium, cut into small pieces, and further disrupted by forcing the tissue through a metal sieve with a sterile plunger to obtain single-cell suspensions. The sieve was rinsed with $5 \mathrm{ml}$ complete RPMI, and cells were centrifuged at $300 \mathrm{~g}$ for 10 minutes at $4^{\circ} \mathrm{C}$.
Cell suspensions were either frozen for future use in $90 \%$ FBS plus $10 \%$ DMSO $\left(1 \times 10^{7}\right.$ cells $\left./ \mathrm{ml}\right)$ or tested for cytokine expression. T cells were distributed into 6 -well plates containing $10^{6}$ cells $/ \mathrm{ml}$ and incubated overnight at $37^{\circ} \mathrm{C}$ in $5 \% \mathrm{CO}_{2}$. The following day, cells were activated with either PBS, HK-GAS, or PMA+I for 6 hours as previously described $(17,18)$. HK-GAS was a mixture of 4 GAS serotypes (M1, M5, M6, and M19) combined in PBS at a ratio of 2:1:1:1, respectively. T cells from all samples were stained and analyzed for CD4, CD8, CD19, IL-17A, and IFN- $\gamma$ by FACS as previously described $(17,18)$. In addition, $\mathrm{T}$ cells from some samples were further analyzed for the memory markers CD45RO, CD45RA, CCR7, and CD62L (eBioscience).

Isolation and analysis of $\mathrm{CD} 4^{+} \mathrm{T}$ cells from mouse brains after multiple GAS infections. The GAS-2W recombinant strain that expresses the $2 \mathrm{~W}$ epitope-tagged $\mathrm{M}$ protein has been described previously (17). HK-GAS-2W streptococci were obtained from log-phase cultures of $2 \times$ $10^{8} \mathrm{CFU}$ bacteria that were resuspended in PBS and heat killed at $60^{\circ} \mathrm{C}$ for 30 minutes. C57BL/6 mice were inoculated with GAS-2W weekly for 4 to 5 weeks. Six or forty-eight hours after the last i.n. inoculation with live bacteria or i.v. injection of HK-GAS, mice were perfused with PBS, then euthanized, and single-cell suspensions of lymphoid and brain tissues were prepared. T cells from brains were purified using a Percoll gradient (63). The T cell response to either i.n. inoculation with GAS or i.v. injection with HK-GAS was characterized with the $2 \mathrm{~W}: \mathrm{I}^{\mathrm{b}} \mathrm{A}^{\mathrm{b}}$ tetramers $(17,24)$. $T$ cell preparations were restimulated ex vivo with PMA+I, stained, and analyzed by FACS as previously described (17). $L M-2 \mathrm{~W}$ (28) and ST-2W (29) have been described in detail in previous studies. The $2 \mathrm{~W}$ epitope is expressed episomally as a secreted protein in $L M-2 \mathrm{~W}$ fused in-frame to the $L M$ hly gene. The $2 \mathrm{~W}$ epitope is tagged chromosomally to the OmpC gene in $S T-2 \mathrm{~W}$.

Differentiation of vasculature versus brain parenchyma $T$ cells. Brain-derived $T$ cells attached to the luminal side of blood vessels were distinguished from those present in the parenchyma as described previously (27). Briefly, mice were injected via the tail vein with a total of $3 \mu$ g anti-mouse CD90.2-APC-eF780 Ab(clone: 53-2.1; eBioscience). Mice were sacrificed 3 minutes after injection of the $\mathrm{Ab}$, perfused with cold PBS for 5 minutes, and tissues harvested and processed for analysis. $\mathrm{CD}^{+} \mathrm{T}$ cells from blood were CD $90.2^{+}$, but those from the parenchyma were not labeled with this $\mathrm{Ab}$ (27).

Flow cytometry and Abs. The following Abs were used for FACS from eBioscience: eFlour 450-conjugated anti-B220 (clone RA3-6B2), CD11b (clone M1/70) and CD8a (clone 53-6.7); PerCP-conjugated CD4 (clone RM4-5); PE-Cy7-conjugated IFN- $\gamma$; PE-conjugated IL-17A; APC-AF780-conjugated CD4 and Alexa Fluor 700-conjugated CD44; PE-conjugated CD4; FITC-conjugated CD4; eFlour 450-conjugated CD4; APC-conjugated CD4; AF700-conjugated CD4; PerCP-Cy5.5conjugated CD4; APC-eF780-conjugated CD4; anti-mouse CCR6 and anti-mouse CD11a (LFA-1); anti-mouse $\alpha 4 \beta 7$ integrin; anti-mouse CD45.2; APC-eF780 (clone 104); and anti-mouse CD45.1 PE (clone A20). FACS acquisition was performed using FACSDiva software, and analysis was done with FlowJo software. A BD LSR II Flow Cytometer was used to analyze cells.

Immunofluorescence. Brains were dissected from C57BL/6J or $\mathrm{SJL} / \mathrm{J}$ mice after PBS perfusion. Tissues were fresh frozen in Tissue-Tek O.C.T., and fresh sections were fixed with $95 \%$ ethanol for 30 minutes, followed by acetone for 1 minute for TJ protein analysis. Immunofluorescence for TJ proteins was performed with Abs for claudin-5 (1:500; Life Technologies); ZO-1 (1:500; Life Technologies); 
and BSL-rhodamine (1:250; Vector Laboratories) as described previously (30). Tissues were fixed for 6 hours with $4 \%$ paraformaldehyde in PBS, cryoprotected, and sectioned for analysis of T cell, microglia, and BBB markers. Sections were stained with Abs against caveolin 1 (1:1000; Abcam); Glut1 (1:2,000; EMD Millipore); CD4 (clone RM4-5, 1:100; BD Pharmingen); CD68 (1:500 Abcam); OMP (1:2,000; Wako Chemicals); Iba1 (1:500; Wako Chemicals); and BSL-FITC or BSLrhodamine (1:250; Vector Laboratories) as previously described (30). Images were acquired with an LSM700 confocal microscope from the Optical Biology Core of the University of California, Irvine, or Columbia University and processed with Fiji software (ImageJ, NIH).

Quantification of activated microglia and synaptic proteins. $\mathrm{CD}^{+} 8^{+} \mathrm{Iba1}^{+}$cells (activated microglia) in the glomerular layer of the OB were quantified using Fiji software. For each animal, all microglia in the glomerular layer were counted in three sections that corresponded approximately to bregma $4.5,4.28$, and 3.56. The values were averaged for each brain. Brain means were then averaged to obtain a mean of means for each treatment group. For synaptic protein quantification, OB sections (12- $\mu \mathrm{m}$ thick) from PBS- and GAS-treated animals were stained for the following synaptic markers: mouse antiGAD67 (1:2,000; EMD Millipore; catalog MAB5406); guinea pig antivGluT2 (1:3,000; EMD Millipore; catalog AB2251); and mouse antiPSD95 (1:1,000; EMD Millipore; catalog MAB1596). Three images were taken at the same bregma point for each animal in order to quantify the medial, ventral, and lateral regions of the OB. Glomeruli were outlined manually, and the mean and SEM fluorescence intensities within the glomerular area were quantified for each channel using Fiji software and averaged to yield a single value for each animal.

Assessment of BBB permeability (biocytin-TMR and IgG leakage quantitation). GAS-inoculated or control mice were injected i.v. via the tail vein with $100 \mu \mathrm{l}$ 1\% biocytin-TMR (catalog T-12921; Life Technologies) either 6 or 48 hours after the last GAS inoculation. Serial brain sections were analyzed for the presence of biocytin-TMR, mouse IgG, and the vascular markers Glut1 (1:2,000; EMD Millipore) and caveolin 1 (1:1,000 Abcam), and biocytin-TMR and IgG leakage was quantified with Fiji software as described previously (30).

TJ quantitation. The total number and fraction of TJs with gaps or protrusions were quantified from confocal microscopic images as previously described (30). Protrusions were identified as bulbous extensions protruding from linear TJ strands. Gaps were identified as TJ strand discontinuities of greater than 1 to $2 \mu \mathrm{m}$ in length. Maximum intensity projections were created in Fiji software for each image $Z$-stack. The number of protrusions and gaps was determined in the resulting 2D projections for each group. Blood vessel borders were defined with the BSL-rhodamine stain.

Statistics. Statistical analysis of mean differences between GASactivated and nonactivated $\mathrm{T}$ cells in tonsils was performed with the nonparametric Wilcoxon matched-pairs signed-rank test. Analysis of statistical differences between naive and GAS-inoculated mice for BBB leakage (biocytin-TMR and IgG leakage) was performed with a 2-tailed Student's $t$ test. A $P$ value of less than 0.05 was considered statistically significant. Analysis of differences in $\mathrm{CD}^{+} \mathrm{T}$ cell distribution in brain regions of naive mice and those of mice that received single or multiple GAS infections or multiple i.n. infections with HK-GAS-2W, HK-LM-2W, or HK-ST-2W was performed with either 1-way ANOVA with Tukey's post-hoc multiple comparisons test or 2-way ANOVA with Bonferroni's post-hoc correction. Differences were considered significant when $P$ values were less than 0.05. Comparison of the levels of various synaptic proteins among naive and GAS-inoculated mice in the $\mathrm{OB}$ or $\mathrm{TJ}$ abnormalities among naive mice and singly and multiply GAS-inoculated mice was performed with 1-way ANOVA with Tukey's post-hoc multiple comparisons test.

Study approval. All animal experiments were conducted with the approval of the IACUCs of the University of Minnesota, the University of California, Irvine, and Columbia University Medical Center. Use of pathological human tonsil tissue waste was determined to be exempt from further review by the Human Subjects Review Committee of the University of Minnesota.

\section{Author contributions}

TD, EDS, DA, and PPC designed all experiments for the study. TD, EDS, DK, MH, MP, and DA performed the experiments. PBE, BC, and PS developed methods for analysis of T cells in human tonsil tissues. EL and EH provided human tonsil tissues. TD, DK, MP, EDS, MH, and DA prepared the figures. TD, DA, EDS, and PPC wrote the manuscript.

\section{Acknowledgments}

We thank Holly and Mark Kerslake from Newport Equities LLC for their generous financial support that made much of this work possible; Ilir Agalliu of the Albert Einstein College of Medicine for help with statistical analysis; Tyler Cutforth for advice on the olfactory system anatomy and olfactory circuitry synapses and for providing critical scientific and editorial input regarding the manuscript; Marc Jenkins for providing 2W:I-A $\mathrm{A}^{\mathrm{b}}$ tetramers; Vaiva Vezys for guidance with the isolation of brain T cells; and Edward Kaplan for suggesting the cribriform plate as a possible window for T cell entry into the brain. E.D. Smith, D. Knowland, M. Hsu, M. Platt, and D. Agalliu are supported by grants from the National Heart, Lung, and Blood Institute (NHLBI), NIH (7R01 HL116995-01), the National Multiple Sclerosis Society (NMSS) (RG4673A1/1), and by a generous donation from Newport Equities LLC. Portions of this work were funded by pandasnetwork.org and by P.P. Cleary, from a royalty account at the University of Minnesota. The confocal facility in the Optical Biology Shared Resource at the University of California, Irvine, was funded by a Cancer Center Support Grant (CA-62203).

Address correspondence to: Dritan Agalliu, Departments of Neurology, Pathology and Cell Biology and Pharmacology, Columbia University Medical Center, 650 West $168^{\text {th }}$ Street, Room 310, New York, New York 10032, USA. Phone: 212.305.0323; E-mail: da191@cumc.columbia.edu. Or to: P. Patrick Cleary, Department of Microbiology and Immunology, University of Minnesota, 420 Delaware Street S.E., MMC 196, Minneapolis, Minnesota 55455, USA. Phone: 651.482.0107; E-mail: clear001@umn.edu.
1. Cunningham MW. Streptococcus and rheumatic fever. Curr Opin Rheumatol. 2012;24(4):408-416.

2. Cardoso F, Eduardo C, Silva AP, Mota CC. Chorea in fifty consecutive patients with rheumatic fever. Mov Disord. 1997;12(5):701-703.

3. Swedo SE. Sydenham's chorea. A model for childhood autoimmune neuropsychiatric disorders. JAMA. 1994;272(22):1788-1791.
4. Murphy TK, Kurlan R, Leckman J. The immunobiology of Tourette's disorder, pediatric autoimmune neuropsychiatric disorders associated with Streptococcus, and related disorders: a 
way forward. JChild Adolesc Psychopharmacol. 2010;20(4):317-331.

5. Murphy TK, Storch EA, Lewin AB, Edge PJ, Goodman WK. Clinical factors associated with pediatric autoimmune neuropsychiatric disorders associated with streptococcal infections. J Pediatr. 2012;160(2):314-319.

6. Swedo SE. Pediatric autoimmune neuropsychiatric disorders associated with streptococcal infections (PANDAS). Mol Psychiatry. 2002; 7(suppl 2):S24-S25.

7. Murphy TK, Gerardi DM, Leckman JF. Pediatric acute-onset neuropsychiatric syndrome. Psychiatr Clin North Am. 2014;37(3):353-374.

8. Kirvan CA, Swedo SE, Snider LA, Cunningham MW. Antibody-mediated neuronal cell signaling in behavior and movement disorders. J Neuroimmunol. 2006;179(1-2):173-179.

9. Dale RC, et al. Antibodies to surface dopamine-2 receptor in autoimmune movement and psychiatric disorders. Brain. 2012;135(pt 11):3453-3468.

10. Murphy TK, et al. Relationship of movements and behaviors to Group A Streptococcus infections in elementary school children. Biol Psychiatry. 2007;61(3):279-284.

11. Wakim LM, et al. The molecular signature of tissue resident memory CD8 $\mathrm{T}$ cells isolated from the brain. J Immunol. 2012;189(7):3462-3471.

12. Ransohoff RM, Engelhardt B. The anatomical and cellular basis of immune surveillance in the central nervous system. Nat Rev Immunol. 2012;12(9):623-635.

13. Yaddanapudi K, et al. Passive transfer of streptococcus-induced Abs reproduces behavioral disturbances in a mouse model of pediatric autoimmune neuropsychiatric disorders associated with streptococcal infection. Mol Psychiatry. 2010;15(7):712-726.

14. Brimberg L, et al. Behavioral, pharmacological, and immunological abnormalities after streptococcal exposure: a novel rat model of Sydenham chorea and related neuropsychiatric disorders. Neuropsychopharmacology. 2012;37(9):2076-2087.

15. Stromnes IM, Goverman JM. Active induction of experimental allergic encephalomyelitis. Nat Protoc. 2006;1(4):1810-1819.

16. Park HS, Francis KP, Yu J, Cleary PP. Membranous cells in nasal-associated lymphoid tissue: a portal of entry for the respiratory mucosal pathogen group A streptococcus. Jimmunol. 2003;171(5):2532-2537.

17. Dileepan T, Linehan JL, Moon JJ, Pepper M, Jenkins MK, Cleary PP. Robust antigen specific th17 T cell response to group a streptococcus is dependent on IL- 6 and intranasal route of infection. PLoS Pathog. 2011;7(9):e1002252.

18. Wang B, et al. Induction of TGF- $\beta 1$ and TGF- $\beta 1$-dependent predominant Th17 differentiation by group A streptococcal infection. Proc Natl Acad Sci U S A. 2010;107(13):5937-5942.

19. Kebir H, et al. Human TH17 lymphocytes promote blood-brain barrier disruption and central nervous system inflammation. Nat Med. 2007;13(10):1173-1175.

20. Huppert J, et al. Cellular mechanisms of IL-17induced blood-brain barrier disruption. FASEB J. 2010;24(4):1023-1034.

21. Alvarez JI, Cayrol R, Prat A. Disruption of central nervous system barriers in multiple sclerosis. Biochim Biophys Acta. 2011;1812(2):252-264.

22. Lim HW, Lee J, Hillsamer P, Kim CH. Human Th17 cells share major trafficking receptors with both polarized effector $\mathrm{T}$ cells and $\mathrm{FOXP}^{+}$regulatory T cells. J Immunol. 2008;180(1):122-129.

23. Lintges $\mathrm{M}$, et al. A new closed-tube multiplex real-time PCR to detect eleven superantigens of Streptococcus pyogenes identifies a strain without superantigen activity. Int J Med Microbiol. 2007;297(6):471-478.

24. Moon JJ, et al. Tracking epitope-specific T cells. Nat Protoc. 2009;4(4):565-581.

25. Owens T, Bechmann I, Engelhardt B. Perivascular spaces and the two steps to neuroinflammation. J Neuropathol Exp Neurol. 2008;67(12):1113-1121.

26. Nagayama S, et al. Differential axonal projection of mitral and tufted cells in the mouse main olfactory system. Front Neural Circuits. 2010;4:120.

27. Anderson KG, et al. Cutting edge: intravascular staining redefines lung CD8 $\mathrm{T}$ cell responses. J Immunol. 2012;189(6):2702-2706.

28. Ertelt JM, Rowe JH, Johanns TM, Lai JC, McLachlan JB, Way SS. Selective priming and expansion of antigen-specific Foxp3 ${ }^{-} \mathrm{CD} 4^{+} \mathrm{T}$ cells during Listeria monocytogenes infection. JImmunol. 2009;182(5):3032-3038.

29. Nelson RW, McLachlan JB, Kurtz JR, Jenkins $\mathrm{MK} . \mathrm{CD} 4^{+} \mathrm{T}$ cell persistence and function after infection are maintained by low-level peptide:MHC class II presentation. J Immunol. 2013;190(6):2828-2834.

30. Knowland D, et al. Stepwise recruitment of transcellular and paracellular pathways underlies blood-brain barrier breakdown in stroke. Neuron . 2014;82(3):603-617.

31. Huerta PT, Kowal C, DeGiorgio LA, Volpe BT, Diamond B. Immunity and behavior: antibodies alter emotion. Proc Natl Acad Sci U S A. 2006;103(3):678-683.

32. Zimmermann J, Krauthausen M, Hofer MJ, Heneka MT, Campbell IL, Muller M. CNStargeted production of IL-17A induces glial activation, microvascular pathology and enhances the neuroinflammatory response to systemic endotoxemia. PLoS One. 2013;8(2):e57307.

33. Murphy AC, Lalor SJ, Lynch MA, Mills KH. Infiltration of Th1 and Th17 cells and activation of microglia in the CNS during the course of experimental autoimmune encephalomyelitis. Brain Behav Immun. 2010;24(4):641-651.

34. Schafer DP, et al. Microglia sculpt postnatal neural circuits in an activity and complement-depen dent manner. Neuron. 2012;74(4):691-705.

35. Ji K, Akgul G, Wollmuth LP, Tsirka SE. Microglia actively regulate the number of functional synapses. PLoS One. 2013;8(2):e56293.

36. Kettenmann H, Kirchhoff F, Verkhratsky A. Microglia: new roles for the synaptic stripper. Neuron. 2013;77(1):10-18.

37. Gabellec MM, Panzanelli P, Sassoe-Pognetto M, Lledo PM. Synapse-specific localization of vesicular glutamate transporters in the rat olfactory bulb. Eur J Neurosci. 2007;25(5):1373-1383.

38. Dale RC, Brilot F. Autoimmune basal ganglia disorders. JChild Neurol. 2012;27(11):1470-1481.
39. Granata T, Cross H, Theodore W, Avanzini G. Immune-mediated epilepsies. Epilepsia. 2012;52(suppl 3):5-11.

40. Goldmann T, Prinz M. Role of microglia in CNS autoimmunity. Clin Dev Immunol. 2013;2013:208093.

41. Rothhammer V, et al. Th17 lymphocytes traffic to the central nervous system independently of $\alpha 4$ integrin expression during EAE. J Exp Med. 2011;208(12):2465-2476.

42. Macedo-Ramos H, et al. Olfactory ensheathing cells as putative host cells for Streptococcus pneumoniae: evidence of bacterial invasion via mannose receptor-mediated endocytosis. Neurosci Res. 2011;69(4):308-313.

43. van Ginkel FW, McGhee JR, Watt JM, CamposTorres A, Parish LA, Briles DE. Pneumococcal carriage results in ganglioside-mediated olfactory tissue infection. Proc Natl Acad Sci U S A. 2003;100(24):14363-14367.

44. Reboldi A, et al. C-C chemokine receptor 6-regulated entry of TH-17 cells into the CNS through the choroid plexus is required for the initiation of EAE. Nat Immunol. 2009;10(5):514-523.

45. Arima Y, et al. Regional neural activation defines a gateway for autoreactive $T$ cells to cross the blood-brain barrier. Cell. 2012;148(3):447-457.

46. Aspelund A, et al. A dural lymphatic vascular system that drains brain interstitial fluid and macromolecules. JExp Med. 2015;212(7):991-999.

47. Louveau A, et al. Structural and functional features of central nervous system lymphatic vessels. Nature. 2015;523(7560):337-341.

48. Broadwell RD, Sofroniew MV. Serum proteins bypass the blood-brain fluid barriers for extracellular entry to the central nervous system. Exp Neurol. 1993;120(2):245-263.

49. Diamond B, Huerta PT, Mina-Osorio P, Kowal C, Volpe BT. Losing your nerves? Maybe it's the antibodies. Nat Rev Immunol. 2009;9(6):449-456.

50. Siffrin $\mathrm{V}$, et al. In vivo imaging of partially reversible th17 cell-induced neuronal dysfunction in the course of encephalomyelitis. Immunity. 2010;33(3):424-436.

51. Marchiando AM, et al. Caveolin-1-dependent occludin endocytosis is required for TNFinduced tight junction regulation in vivo. JCell Biol. 2010;189(1):111-126.

52. Diamond B, Honig G, Mader S, Brimberg L, Volpe BT. Brain-reactive antibodies and disease. Annu Rev Immunol. 2013;31:345-385.

53. Tuomanen EI, et al. Reversible opening of the blood-brain barrier by anti-bacterial antibodies. Proc Natl Acad Sci U S A. 1993;90(16):7824-7828.

54. Bystrom J, Al-Adhoubi N, Al-Bogami M, Jawad AS, Mageed RA. Th17 lymphocytes in respiratory syncytial virus infection. Viruses. 2013;5(3):777-791.

55. Maroof A, Yorgensen YM, Li Y, Evans JT. Intranasal vaccination promotes detrimental Th17-mediated immunity against influenza infection. PLoS Pathog. 2014;10(1):e1003875.

56. Truchetet ME, et al. Potential role of Mycoplasma hominis in interleukin (IL)-17-producing $\mathrm{CD} 4{ }^{+} \mathrm{T}$-cell generation via induction of IL-23 secretion by human dendritic cells. J Infect Dis. 2011;204(11):1796-1805.

57. Frank KM, Zhou T, Moreno-Vinasco L, Hollett B, Garcia JG, Bubeck Wardenburg J. Host response 
signature to Staphylococcus aureus $\alpha$-hemolysin implicates pulmonary Th17 response. Infect Immun. 2012;80(9):3161-3169.

58. Debertin AS, Tschernig T, Tonjes H, Kleemann WJ, Troger HD, Pabst R. Nasal-associated lymphoid tissue (NALT): frequency and localization in young children. Clin Exp Immunol. 2003;134(3):503-507.

59. Nordenankar K, Bergfors A, Wallen-Mackenzie A. Targeted deletion of Vglut 2 expression in the embryonal telencephalon promotes an anxiolytic phenotype of the adult mouse. Ups J Med Sci. 2015;120(3):144-156.

60. Tordera RM, et al. Enhanced anxiety, depressivelike behaviour and impaired recognition memory in mice with reduced expression of the vesicular glutamate transporter 1 (VGLUT1). Eur J Neurosci. 2007;25(1):281-290.

61. Guilherme L, Kalil J. Rheumatic heart disease: molecules involved in valve tissue inflammation leading to the autoimmune process and antiS. pyogenes vaccine. Front Immunol. 2013;4:352.

62. Shulman ST, Stollerman G, Beall B, Dale JB, Tanz RR. Temporal changes in streptococcal M protein types and the near-disappearance of acute rheumatic fever in the United States. Clin Infect Dis. 2006;42(4):441-447.

63. Pino PA, Cardona AE. Isolation of brain and spinal cord mononuclear cells using percoll gradients. JVis Exp. 2011;(48):2348. 